OPEN ACCESS

Edited by:

Angel Lanas,

University of Zaragoza, Spain

Reviewed by:

Zuzana Macek Jilkova

Centre Hospitalier Universitaire de

Grenoble, France

Mengfei Liu,

Mayo Clinic, United States

*Correspondence:

Magda Shaheen

magdashaheen@cdrewu.edu

Specialty section:

This article was submitted to

Gastroenterology,

a section of the journal

Frontiers in Medicine

Received: 15 October 2021 Accepted: 08 November 2021 Published: 02 December 2021

Citation:

Shaheen M, Schrode KM, Pan D,

Kermah D, Puri V, Zarrinpar A, Elisha D, Najjar SM and Friedman TC (2021) Sex-Specific Differences in the Association Between Race/Ethnicity and NAFLD Among US Population.

Front. Med. 8:795421.

doi: 10.3389/fmed.2021.795421

\section{Sex-Specific Differences in the Association Between Race/Ethnicity and NAFLD Among US Population}

\author{
Magda Shaheen ${ }^{1 *}$, Katrina M. Schrode ${ }^{1}$, Deyu Pan ${ }^{1}$, Dulcie Kermah ${ }^{1}$, Vishwajeet Puri ${ }^{2}$, \\ Ali Zarrinpar ${ }^{3}$, David Elisha ${ }^{1}$, Sonia M. Najjar ${ }^{2}$ and Theodore C. Friedman ${ }^{1}$ \\ ${ }^{1}$ College of Medicine, Charles R Drew University, Los Angeles, CA, United States, ${ }^{2}$ Heritage College of Osteopathic \\ Medicine, Ohio University, Athens, OH, United States, ${ }^{3}$ University of Florida College of Medicine, Gainesville, FL, \\ United States
}

Non-alcoholic fatty liver disease (NAFLD) is spreading worldwide, with a racial/ethnic disparity. We examined the gender role in the racial/ethnic difference in NAFLD in the US population. We analyzed data for 3,292 individuals $\geq 18$ years old from NHANES 2017-2018, a representative sample of the non-institutionalized adult population in the US. Exclusions were subjects with elevated transferrin level, chronic hepatitis B or C, excessive alcohol use, or prescription medications that might cause hepatic steatosis. NAFLD was diagnosed by FibroScan ${ }^{\circledR}$ using controlled attenuation parameter (CAP) values: S0 <238, S1 = 238-259, S2 = 260-290, S3 >290. Data were analyzed using Chi square and multinomial regression. The overall prevalence of NAFLD was $47.9 \%$ [S2 = $16.1 \%$, and S3 $=31.8 \%]$. The prevalence of S3 was highest among Mexican Americans (46\%), lowest among Blacks (22.7\%), 29.9\% in other Hispanics and $32.1 \%$ in Whites $(p$ $<0.05)$. It was higher among Mexican American males (54.1\%) compared to Mexican American females (37.7\%) $(p<0.05)$. In the adjusted model, Mexican Americans were two times more likely than Whites to have S2 and S3 $(p<0.05)$. Only male Mexican Americans had higher odds of S2 and S3 relative to male White $(p<0.05)$. Males had higher odds of S3 relative to non-menopausal females $(p<0.05)$. There was no difference in the odds of S2 or S3 NAFLD among the menopausal females with or without hormone therapy relative to non-menopausal females $(p>0.05)$. While Mexican Americans had the highest prevalence of severe NAFLD relative to the other racial/ethnic groups, only male Mexican Americans, but not females, had higher likelihood of both moderate and severe NAFLD relative to Whites. Interventions that specifically target Mexican American males are needed to increase awareness about NAFLD and its prevention.

Keywords: sex, non-alcoholic fatty liver disease (NAFLD), NHANES 2017-2018, race/ethnicity, disparity

\section{INTRODUCTION}

Non-alcoholic fatty liver disease (NAFLD) is a public health concern affecting about 100 million Americans (1), and the prevalence of NAFLD has been increasing (2). In the United States, the prevalence of NAFLD has risen from $18 \%$ in 1988-1991 to 31\% in 2011-2012 (3). NAFLD is the most common form of liver disease and is the second leading reason for liver transplants in the United States $(4,5)$. Due to the impact of NAFLD on the health of individuals, in the United States, the health care cost for NAFLD is estimated to be $\$ 103$ billion (6). There is a range of severity 
associated with NAFLD. The spectrum ranges from hepatic steatosis, to non-alcoholic steatohepatitis (NASH), fibrosis, and cirrhosis (7). NASH is a progressive form of NAFLD and research shows that it could lead to fatal conditions including cirrhosis and hepatocellular carcinoma (8-10). Among individuals classified as having severe obesity, one out of four have NASH (11). Risk factors for developing NAFLD include mainly age, race/ethnicity and metabolic conditions, and NAFLD is also known to be associated with an increased risk with obesity, type 2 diabetes, metabolic syndrome, and cardiovascular diseases (12-14).

Previous studies have shown that there is a racial/ethnic disparity with NAFLD (1). Hispanics are known to have a higher prevalence of NAFLD and blacks a lower prevalence of NAFLD compared to non-Hispanic Whites $(2,15)$. We recently found that Mexican Americans were about 2 times more likely than non-Hispanic Whites to have advanced hepatic steatosis, while other Hispanics showed no difference from Whites (16). In patients with biopsy-proven NAFLD and severe obesity, studies show that Whites were significantly more likely to have NAFLD, NASH and advanced fibrosis compared to blacks (17). The risk for NASH among patients with NAFLD was higher in Hispanics and lower in Blacks. In addition, studies have shown genetic factors contribute to the racial/ethnic differences in NAFLD (1821). Although it has been shown that Whites are more likely to be re-admitted for cirrhosis, an analysis of the Nationwide Inpatient Sample showed that Blacks and Hispanic were less likely to receive liver transplants compared to Whites, and blacks had a higher in-hospital mortality than Whites (22). Moreover, whereas fibrosis has been linked with NAFLD, over time patients remain stable; hence, there is an opportunity to intervene during this time $(23,24)$.

There have been conflicting results regarding the role of gender as a risk factor for NAFLD, with most population-based studies finding a higher prevalence of NAFLD among males (25), although patients with biopsy-proven NASH in the NASH Clinical Research Network are more likely to be females (26). In one of few studies examining both gender and race in NAFLD, Browning et al. (27) found that sex differences existed only among Whites, and the presence of a sex difference also varied between Hispanics of different heritage (28).

The current study aimed to examine the role of gender and menopausal status in the association between racial/ethnic disparity and NAFLD. We hypothesized that there is a gender disparity in NAFLD among American Hispanic population.

\section{RESEARCH DESIGN AND METHODS}

\section{Study Population}

We analyzed data for 3,292 participants 18 years and older using the National Health and Nutrition Examination Survey (NHANES) 2017-2018. NHANES samples the US population using a complex, multistage probability design, and obtains informed consent from all participants. NHANES protocols were approved by the National Center for Health Statistics Research Ethics Review Board. Our analysis of these publicly-available data was exempt from Charles R. Drew University IRB review.

\section{Dependent Variable}

Liver fibrosis was measured by FibroScan $^{\circledR}$ which uses ultrasound and vibration controlled transient elastography (VCTETM) to derive liver stiffness. The device also simultaneously measures the ultrasound attenuation related to the presence of hepatic steatosis and records the controlled attenuation parameter (CAPTM) as the indicator for liver fat. We categorized the steatosis status using the median CAP $\mathrm{dB} / \mathrm{m}$ for steatosis grades whereby S0 (no steatosis) $<238$; S1 (mild steatosis) $=238-259$; S2 (moderate steatosis) $=260-290$; and S3 (severe steatosis) >290. Subjects were considered to have NAFLD if they had hepatic steatosis and did not have any exclusion criteria. Exclusion criteria included elevated transferrin level $>50 \%$, chronic hepatitis $\mathrm{B}$, chronic hepatitis $\mathrm{C}$, excessive alcohol use, or prescription medications that might cause hepatic steatosis. Chronic hepatitis B was defined as positive results for both the hepatitis B surface antigen and hepatitis B core antibody tests. Chronic hepatitis $\mathrm{C}$ was defined as positive results for both the hepatitis $\mathrm{C}$ antibody and RNA tests. Excessive alcohol use was defined as an average of more than 2 drinks/day for men or $1 \mathrm{drink} /$ day for women. Average alcohol use was determined using the responses to the two questions: "how often drank any type of alcoholic beverage" and "average drinks on a day when drank alcohol" to calculate a daily average. We excluded subjects if they were taking any of the following hepatotoxic drugs: corticosteroids, antiarrhythmics, anticancerantimetabolites, anticancer-hormonal drugs, anti-convulsant drugs, or nucleoside/nucleotide reverse transcriptase inhibitors.

\section{Independent Variables and Measures}

In our analyses, we included demographic variables (age, gender, race/ethnicity, education, language spoken, and poverty), menopausal status for women, physical activity status, smoking status, diet quality (healthy eating index), body composition (waist-to-hip ratio and body mass index), and laboratory values [cholesterol, HDL, triglyceride, glucose, hemoglobin A1c (HbAlc), highly-sensitive C-reactive protein (hsCRP), AST, and ALT].

Physical activity was based on activity during work, commuting, or leisure time and categorized into 3 categories based on national guidelines which recommend moderate exercise 5 or more times/week or vigorous exercise 3 times/week [ 0 = inactive (no activity); $1=$ does not meet guidelines; and $2=$ meets guidelines]. Age was categorized as 18-19 years; 20-34 years; $35-49$ years; $50-64$ years, and $\geq 65$. Education was categorized as less than high school ( $<12$ grade), high school (12 grade), some college, and at least college degree. Gender was categorized as male and female. Females were further categorized by menopausal status. They were considered menopausal if they stated they had not had "at least one period in the last 12 months," with menopause given as the reason. The menopausal group was further categorized into those taking or not taking hormone replacements based a yes/no response to the question "Have you ever used female hormones such as estrogen and progesterone? Please include any forms of female hormones, such as pills, cream, patch, and injectable, but do not include birth control methods or use for infertility." Race/ethnicity 
was categorized as non-Hispanic White, non-Hispanic Black, Mexican American, Other Hispanics, and Other race, including multi-racial. Language spoken at home was classified as English, Spanish, both, and other languages. Federal income ratio (FIR) was classified as $<1,1-2$, and $>2$ times the federal poverty level. Smoking status was categorized into non-smoker, former, and current smoker. Participants were classified using body mass index (BMI) with BMI $<25$ (normal), BMI = 25-29.9 (overweight), and BMI $=30$ and higher (obese). Waist-to-hip ratio was classified as risk for women $(\geq 0.85) /$ risk for men $(\geq 1.0)$ vs. healthy. Diet quality using the healthy eating index score was categorized as good quality diet, needs improvement in quality diet, and poor-quality diet. Based on the hemoglobin A1c (HbA1c), subjects were classified as normal (healthy) $(<5.7 \%)$, with pre-diabetes $(5.7-6.4 \%)$, and with diabetes $(6.5 \%$ and higher). Total cholesterol was categorized as normal $(<200$ $\mathrm{mg} / \mathrm{dL}$ ), elevated (200-239 mg/dL), and high ( $\geq 240 \mathrm{mg} / \mathrm{dL})$. High-density lipoprotein (HDL) was categorized as low $(<40$ $\mathrm{mg} / \mathrm{dL})$, borderline $(40-59 \mathrm{mg} / \mathrm{dL})$, and healthy $(\geq 60 \mathrm{mg} / \mathrm{dL})$. Triglyceride level was categorized as normal $(<150 \mathrm{mg} / \mathrm{dL})$, borderline (150-199 mg/dL), and high ( $\geq 200 \mathrm{mg} / \mathrm{dL})$. High sensitivity C-reactive protein (hsCRP) was categorized as normal (0.1-1.0 mg/dL), mild inflammation (1.0-3 mg/dL), significant inflammation $(3-10 \mathrm{mg} / \mathrm{dL})$, and highly significant inflammation $(\geq 10 \mathrm{mg} / \mathrm{dL})$. Alanine aminotransferase (ALT) was categorized as normal $(<56 \mathrm{U} / \mathrm{L})$ and elevated $(\geq 56 \mathrm{U} / \mathrm{L})$; and aspartate aminotransferase (AST) was categorized as normal $(<40 \mathrm{U} / \mathrm{L})$ and elevated $(\geq 40 \mathrm{U} / \mathrm{L})$.

\section{Statistical Analyses}

We used descriptive statistics including unweighted number and weighted percent for categorical variables. Missing data was $12.75 \%$ for FIR and $<8 \%$ for all other variables. Bivariate analysis using Chi-Square test for categorical variables were used to determine the statistical difference between the racial/ethnic groups and the other independent variables in the prevalence of NAFLD. We performed multinomial regression analysis with listwise deletion to determine the racial/ethnic difference as well as the associated factors of NAFLD stages adjusting for the confounding variables. In order to examine the role of gender in the relationship between race/ethnicity and NAFLD, we conducted a stratified analysis multinomial regression analysis for males and females. The data are presented as adjusted odds ratio and $95 \%$ confidence interval. $P$-value of $<0.05$ was considered statistically significant. The data were analyzed using SAS (Release V.9. 3, 2002; SAS, Inc.). We used the sample weights provided by the NCHS to correct for differential selection probabilities and to adjust for non-coverage and non-response. All estimates were weighted as supplied by NHANES, and the design is being taken into consideration.

\section{RESULTS}

\section{Population Characteristics}

Of the 3,292 subjects in our sample from NHANES 20172018, 27.6\% were 50-64 years of age and 18.2\% were 65 years and older; 9.9\% were Black, $8.5 \%$ were Mexican Americans,
TABLE 1 | Population characteristics by NAFLD status, NHANES 2017-2018.

\begin{tabular}{|c|c|c|c|c|c|}
\hline & \multirow[t]{2}{*}{ Overall } & \multicolumn{4}{|c|}{ NAFLD } \\
\hline & & $\begin{array}{c}\text { No/mild } \\
(<260)\end{array}$ & $\begin{array}{l}\text { Moderate } \\
(260-290)\end{array}$ & $\begin{array}{l}\text { Severe } \\
(>290)\end{array}$ & $p$-value \\
\hline Overall & 3,292 & 1,668 (52.1\%) & 539 (16.1\%) & $\begin{array}{c}1,085 \\
(31.8 \%)\end{array}$ & \\
\hline Race/ethnicity & & & & & $<0.0001$ \\
\hline Mexican American & $441(8.5)$ & 157 (36.9) & 75 (17.2) & 209 (46.0) & \\
\hline Other Hispanic & $286(6.0)$ & $147(56.4)$ & $44(13.8)$ & 95 (29.9) & \\
\hline $\begin{array}{l}\text { Non-Hispanic } \\
\text { White }\end{array}$ & $1,220(65.6)$ & 605 (52.4) & $194(15.5)$ & $421(32.1)$ & \\
\hline $\begin{array}{l}\text { Non-Hispanic } \\
\text { Black }\end{array}$ & 718 (9.9) & $424(61.2)$ & $115(16.1)$ & $179(22.7)$ & \\
\hline Other race & 627 (9.9) & 335 (51.6) & $111(20.6)$ & $181(27.8)$ & \\
\hline Age (years) & & & & & $<0.0001$ \\
\hline $18-19$ & 165 (3.3) & $130(83.7)$ & $10(5.5)$ & $25(10.8)$ & \\
\hline 20-34 & 742 (27.4) & $479(65.8)$ & 97 (11.5) & $166(22.7)$ & \\
\hline 35-49 & 728 (23.6) & $352(51.1)$ & $124(17.4)$ & 252 (31.5) & \\
\hline 50-64 & 922 (27.6) & 377 (42.1) & $173(18.7)$ & $372(39.2)$ & \\
\hline $65+$ & 735 (18.2) & $330(42.4)$ & 135 (19.3) & $270(38.4)$ & \\
\hline Sex & & & & & 0.0016 \\
\hline Male & 1,596 (48.2) & 745 (47.5) & 254 (16.0) & $597(36.5)$ & \\
\hline Female & 1,696 (51.8) & $923(56.4)$ & 285 (16.2) & 488 (27.4) & \\
\hline
\end{tabular}

\section{Education}

0.0036

Less than high

school

High school

Some college

At least college

degree

Language

0.1064

spoken at home

English

Spanish

Both

Other

$576(9.9) \quad 286(50.5)$

$95(19.7) \quad 195(29.9)$

$821(28.1) \quad 421(50.6)$

$120(13.9) \quad 280(35.5)$

$1,097(30.9) \quad 536(49.2) \quad 180(15.1) \quad 381(35.7)$

$798(31.1) \quad 425(57.0) \quad 144(17.9) \quad 229(25.1)$

Federal income ratio (FIR)

$<1$

$1-2$

$>2$

2,35

$2,358(82.9) \quad 1,244(53.2)$

$376(16.0) \quad 738(30.8)$

$40(17.4) \quad 96(43.4)$

$\begin{array}{llll}357(7.4) & 155(47.5) & 53(14.2) & 149(38.3) \\ 358(6.2) & 186(50.5) & 70(18.4) & 102(31.0)\end{array}$

\section{Waist-hip ratio}

Healthy

$358(6.2)-186(50.5)$

0.3495

Risk for women

$(\geq 0.85) /$ risk for

men ( $\geq 1.0)$

Body mass index

$616(12.4) \quad 345(57.4) \quad 80(13.2) \quad 191(29.4)$

$890(19.6) \quad 435(51.7) \quad 155(18.0) \quad 300(30.3)$

$1,786(68.0) \quad 888(51.3) \quad 304(16.1) \quad 594(32.6)$

$1,442(46.3) \quad 966(70.3) \quad 203(12.1) \quad 273(17.6)$

$1,850(53.7) \quad 702(36.5) \quad 336(19.5) \quad 812(44.0)$

$<0.0001$

(BMI)

Normal or healthy

$(<25)$

Overweight

$(25-<30)$

Obese $(\geq 30)$

$1,307(41.0) \quad 331(25.7)$

$248(18.9) \quad 728(55.5)$
$<0.0001$ 
TABLE 1 | Continued

\begin{tabular}{|c|c|c|c|c|c|}
\hline & \multirow[t]{2}{*}{ Overall } & \multicolumn{4}{|c|}{ NAFLD } \\
\hline & & $\begin{array}{c}\text { No/mild } \\
(<260)\end{array}$ & $\begin{array}{l}\text { Moderate } \\
(260-290)\end{array}$ & $\begin{array}{l}\text { Severe } \\
(>290)\end{array}$ & $p$-value \\
\hline Current & 550 (15.6) & $333(60.2)$ & 70 (14.9) & 147 (24.9) & \\
\hline Former & 758 (24.3) & $302(41.6)$ & $137(18.8)$ & 319 (39.6) & \\
\hline Non-smoker & $1,984(60.0)$ & 1,033 (54.3) & 332 (15.3) & $619(30.4)$ & \\
\hline Alcohol use & & & & & 0.1039 \\
\hline Current drinker & 2,248 (76.3) & 1,159 (53.3) & $372(16.2)$ & $717(30.5)$ & \\
\hline Former drinker & 642 (16.1) & 295 (47.5) & 109 (18.9) & 238 (33.6) & \\
\hline Never drank & 339 (7.6) & $180(50.7)$ & $50(10.8)$ & $109(38.5)$ & \\
\hline Physical activity & & & & & $<0.0001$ \\
\hline Inactive & 733 (18.5) & $318(43.9)$ & 133 (17.6) & $282(38.5)$ & \\
\hline $\begin{array}{l}\text { Does not meet } \\
\text { guideline }\end{array}$ & 515 (15.2) & $241(43.2)$ & 77 (14.6) & $197(42.2)$ & \\
\hline Meets guidelines & 2,044 (66.2) & 1,109 (56.5) & $329(16.0)$ & $606(27.5)$ & \\
\hline $\begin{array}{l}\text { Healthy eating } \\
\text { index }\end{array}$ & & & & & 0.0110 \\
\hline Poor diet & 2,304 (71.4) & $1,157(49.8)$ & 379 (16.8) & 768 (33.5) & \\
\hline $\begin{array}{l}\text { Needs } \\
\text { improvement }\end{array}$ & 887 (26.0) & $460(56.8)$ & $140(14.7)$ & 287 (28.5) & \\
\hline Good diet & $101(2.6)$ & $51(70.0)$ & $20(10.9)$ & $30(19.1)$ & \\
\hline $\begin{array}{l}\text { Serum } \\
\text { cholesterol }\end{array}$ & & & & & 0.0112 \\
\hline $\begin{array}{l}\text { Good (<200 } \\
\text { mg/dL) }\end{array}$ & $2,137(62.7)$ & $1,143(55.2)$ & 330 (15.7) & 664 (29.1) & \\
\hline $\begin{array}{l}\text { Elevated (200-239 } \\
\mathrm{mg} / \mathrm{dL})\end{array}$ & 825 (27.1) & $384(47.7)$ & $147(16.4)$ & 294 (35.9) & \\
\hline $\begin{array}{l}\operatorname{High}(\geq 240 \\
\text { mg/dL) }\end{array}$ & $330(10.2)$ & $141(45.0)$ & $62(17.7)$ & $127(37.4)$ & \\
\hline $\begin{array}{l}\text { High-density } \\
\text { lipoproteins }\end{array}$ & & & & & $<0.0001$ \\
\hline Low (<40 mg/dL) & 556 (15.4) & $157(28.4)$ & 91 (15.3) & 308 (56.3) & \\
\hline $\begin{array}{l}\text { Borderline risk } \\
\text { (40-59 mg/dL) }\end{array}$ & $1,785(54.5)$ & $853(48.3)$ & 301 (16.9) & $631(34.8)$ & \\
\hline $\begin{array}{l}\text { Healthy ( } \geq 60 \\
\mathrm{mg} / \mathrm{dL} \text { ) }\end{array}$ & $951(30.1)$ & $658(71.2)$ & $147(15.0)$ & $146(13.7)$ & \\
\hline $\begin{array}{l}\text { Serum } \\
\text { triglycerides }\end{array}$ & & & & & $<0.0001$ \\
\hline $\begin{array}{l}\text { Normal (<150 } \\
\mathrm{mg} / \mathrm{dL})\end{array}$ & 2,236 (67.5) & 1,370 (63.6) & $339(14.9)$ & $527(21.5)$ & \\
\hline $\begin{array}{l}\text { Borderline } \\
\text { (150-199 mg/dL) }\end{array}$ & $505(15.9)$ & $165(34.1)$ & $113(22.6)$ & $227(43.2)$ & \\
\hline $\begin{array}{l}\operatorname{High}(\geq 200 \\
\text { mg/dL) }\end{array}$ & $551(16.6)$ & $133(22.8)$ & 87 (14.6) & 331 (62.5) & \\
\hline \multicolumn{6}{|l|}{$\begin{array}{l}\text { High-Sensitivity } \\
\text { CRP }\end{array}$} \\
\hline $\begin{array}{l}\text { Normal }(0.1-<1 \\
\mathrm{mg} / \mathrm{dL})\end{array}$ & 1,004 (31.2) & 705 (71.9) & $137(12.4)$ & 162 (15.6) & $<0.0001$ \\
\hline $\begin{array}{l}\text { Mild inflammation } \\
(1-<3 \mathrm{mg} / \mathrm{dL})\end{array}$ & 1,171 (36.2) & 559 (49.1) & 217 (19.7) & 395 (31.2) & \\
\hline $\begin{array}{l}\text { Significant } \\
\text { inflammation } \\
(3-<10 \mathrm{mg} / \mathrm{dL})\end{array}$ & 884 (25.9) & 328 (37.7) & $144(15.6)$ & $412(46.7)$ & \\
\hline
\end{tabular}

TABLE 1 | Continued

\begin{tabular}{|c|c|c|c|c|c|}
\hline & \multirow[t]{2}{*}{ Overall } & \multicolumn{4}{|c|}{ NAFLD } \\
\hline & & $\begin{array}{l}\text { No/mild } \\
(<260)\end{array}$ & $\begin{array}{l}\text { Moderate } \\
(260-290)\end{array}$ & $\begin{array}{l}\text { Severe } \\
(>290)\end{array}$ & $p$-value \\
\hline $\begin{array}{l}\text { High significant } \\
\text { inflammation ( } \geq 10 \\
\mathrm{mg} / \mathrm{dL} \text { ) }\end{array}$ & $233(6.7)$ & 76 (31.9) & $41(15.7)$ & $116(52.4)$ & 0.0367 \\
\hline \multicolumn{6}{|l|}{$\begin{array}{l}\text { Aspartate } \\
\text { aminotransferase } \\
\text { (AST) }\end{array}$} \\
\hline Normal ( $\leq 40$ U/L) & $3,165(96.1)$ & $1,617(52.6)$ & 524 (16.2) & $\begin{array}{l}1,024 \\
(31.1)\end{array}$ & \\
\hline Elevated (>40 U/L) & 127 (3.9) & $51(39.4)$ & 15 (12.5) & $61(48.1)$ & \\
\hline $\begin{array}{l}\text { Alanine } \\
\text { aminotransferase } \\
\text { (ALT) }\end{array}$ & & & & & 0.0031 \\
\hline Normal ( $\leq 56$ U/L) & 3,179 (96.4) & $1,634(52.8)$ & 526 (16.2) & $\begin{array}{l}1,019 \\
(31.0)\end{array}$ & \\
\hline Elevated (>56 U/L) & $113(3.6)$ & 34 (33.2) & $13(13.0)$ & $66(53.7)$ & \\
\hline $\begin{array}{l}\text { Hemoglobin A1c } \\
\text { (HbA1c) }\end{array}$ & & & & & $<0.0001$ \\
\hline Healthy $(<5.7 \%)$ & 1,902 (67.2) & $1,194(62.5)$ & $287(15.8)$ & $421(21.7)$ & \\
\hline $\begin{array}{l}\text { Pre-diabetes } \\
(5.7-6.4 \%)\end{array}$ & 953 (23.8) & 378 (36.4) & 185 (18.0) & 390 (45.5) & \\
\hline Diabetes ( $\geq 6.5 \%$ ) & $437(9.0)$ & 96 (16.2) & 67 (13.1) & $274(70.6)$ & \\
\hline
\end{tabular}

and $6 \%$ were other Hispanics. About half of the population were male (48.2\%), 9.9\% had less than high-school education, and $12.4 \%$ were poor (FIR $<1$ ). Most of the participants (82.9\%) spoke English at home, 15.6\% were current smokers, $18.5 \%$ were physically-inactive (did no exercise), $71.4 \%$ had poor diet, $53.7 \%$ had high waist-to-hip ratio $(\geq 0.85$ for males, $\geq 0.9$ for females) and $41 \%$ were obese by BMI. $10.2 \%$ had high total cholesterol $(\geq 240 \mathrm{mg} / \mathrm{dL}), 15.4 \%$ had low HDL, $16.6 \%$ had high level of triglyceride $(\geq 200 \mathrm{mg} / \mathrm{dL})$, and $32.6 \%$ had significant inflammation as indicated by $>3$ $\mathrm{mg} / \mathrm{dL}$ hsCRP level, 3.9\% had abnormal AST, 3.6\% had abnormal ALT, $23.8 \%$ had pre-diabetes, and $9 \%$ had diabetes (Table 1).

\section{Prevalence of NAFLD Stages}

Overall, 52.1\% had no-to-mild NAFLD, $16.1 \%$ had moderate NAFLD, and $31.8 \%$ had severe NAFLD (Table 1). The prevalence of NAFLD varied significantly by all the independent variables $(p<0.05)$ except the language spoken at home, FIR, and alcohol use $(p>0.05)$. The highest prevalence of moderate NAFLD was among subjects 65 years and older (19.3\%) and that of severe NAFLD was among 50-64 years old $(39.2 \%)$ ( $p$ $<0.05)$. More than one third of males $(36.5 \%)$ had severe NAFLD compared to $27.4 \%$ among females $(p<0.05)$. The highest prevalence of moderate NAFLD was among the other racial/ethnic group (20.6\%) and that of severe NAFLD was among Mexican Americans (46.0\%) and the lowest prevalence was among Black population $(22.7 \%)(p<0.05$; Figure 1). The highest prevalence of moderate NAFLD was among participants 


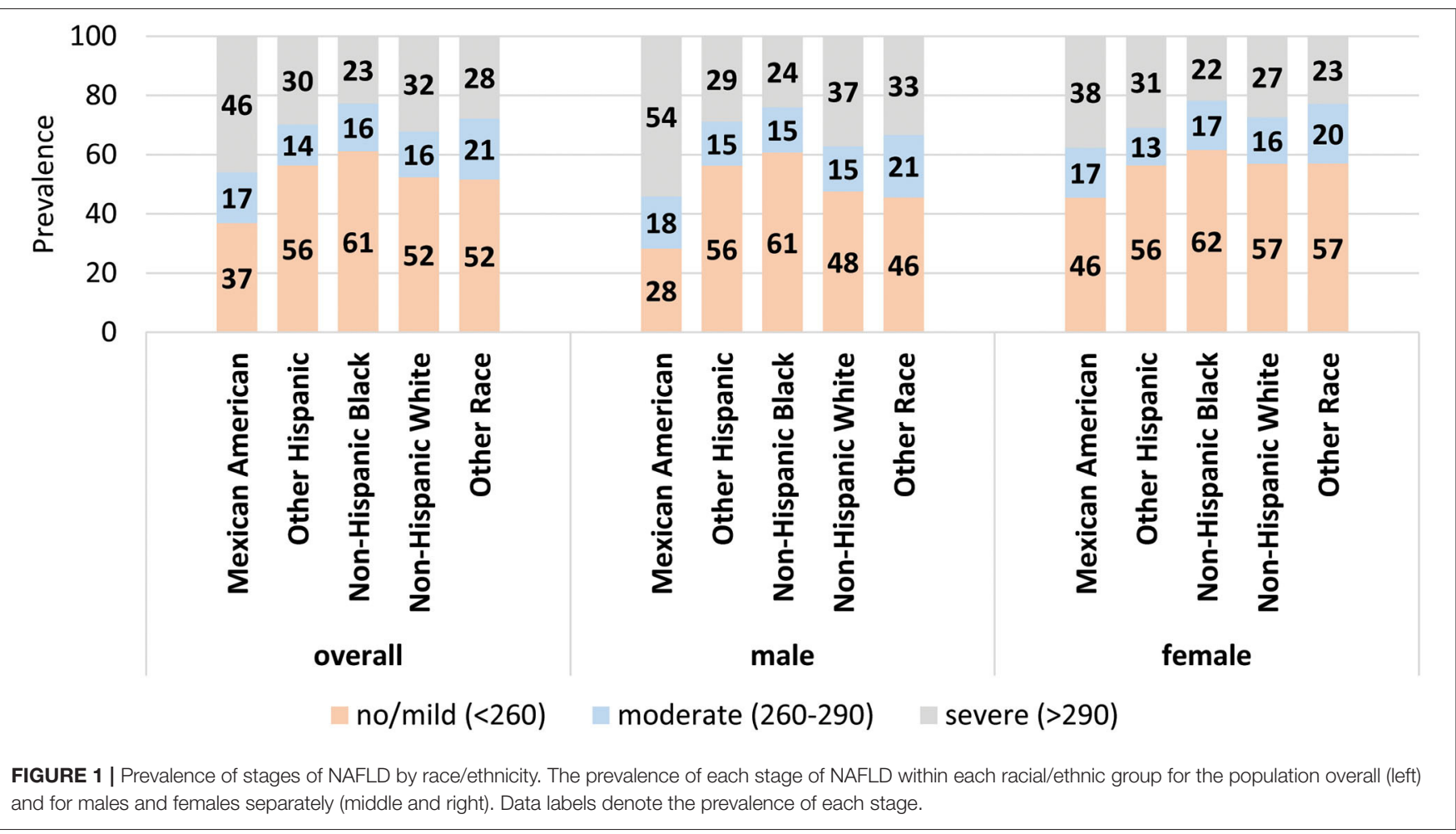

with less than high school education and of severe NAFLD was among those with some college education (35.7\%) $(p<0.05)$.

Forty-four percent of subjects with high waist-to-hip ratio had severe NAFLD $(p<0.05)$. The highest prevalence of moderate NAFLD was among the overweight group (20.7\%) and the highest prevalence of severe NAFLD was among the obese group (55.5\%) $(p<0.05)$. Former smokers had the highest prevalence of moderate NAFLD (18.8\%) and severe NAFLD (39.6\%) $(p<0.05)$. About $17.6 \%$ of the physically inactive subjects had moderate NAFLD and $42.2 \%$ has severe NAFLD $(p<0.05)$. Subjects who consumed a poor-quality diet had the highest prevalence of moderate $(16.8 \%)$ and severe NAFLD (33.5\%). Of the subjects with high cholesterol level, $17.7 \%$ had moderate NAFLD and $37.4 \%$ had severe NAFLD $(p<0.05)$. Of those with borderline triglyceride levels, $22.6 \%$ had moderate NAFLD and $43.2 \%$ had severe NAFLD, while among those with high triglycerides, $62.5 \%$ had severe NAFLD $(p<0.05)$. The borderline HDL group had $34.8 \%$ prevalence and the low HDL group had a $56.3 \%$ prevalence of severe NAFLD. The highest prevalence of moderate NAFLD was among those with mild inflammation (19.7\%) and the highest prevalence of severe NAFLD was among those with hsCRP level $\geq 10 \mathrm{mg} / \mathrm{dL}(52.4 \%)(p<0.05)$. As expected, the highest prevalence of severe NAFLD was among those with elevated AST (48.1\%) and ALT (53.7\%) $(p<0.05)$. In the context of diabetes, moderate NAFLD was more prevalent among prediabetes population $(18.0 \%)$ while diabetic population had a $70.6 \%$ prevalence of severe NAFLD ( $p<0.05$; Table 1$)$.

Table 2 shows the prevalence of NAFLD stages by the independent variables among males and females. Among males, a significantly higher prevalence of moderate NAFLD was found among "other race" group (Figure 1), 65 years and older, with less than high school education, former smokers, overweight, had high waist-to-hip ratio, did not meet the guidelines for physical activity, but had cholesterol levels $<200 \mathrm{mg} / \mathrm{dL}, 40-59 \mathrm{mg} / \mathrm{dL}$ HDL, triglyceride levels of 150-199 mg/dL, 1-<3 mg/dL CRP levels, and HbAlc levels of 5.7-6.4\% $(p<0.05)$. The higher prevalence of severe NAFLD was among Mexican Americans (Figure 1), age 50-65 years of age, had some college education, obese, former smokers, physically inactive, had high waist-tohip ratio, cholesterol levels $>240 \mathrm{mg} / \mathrm{dL}, \mathrm{HDL}<40 \mathrm{mg} / \mathrm{dL}$, triglyceride $>200 \mathrm{mg} / \mathrm{dL}, \mathrm{CRP}>10 \mathrm{mg} / \mathrm{dL}, \mathrm{ALT}>56 \mathrm{U} / \mathrm{L}$, and HbA1c $>6.5 \%(p<0.05)$.

Among females, significantly higher prevalence of moderate NAFLD was found among 65 years and older, with less than high school education, current smokers, obese, had high waist-to-hip ratio, physically inactive, ate poor-quality diet, had HDL 40-59 $\mathrm{mg} / \mathrm{dL}$, triglyceride levels of 150-199 mg/dL, CRP levels of 1$<3 \mathrm{mg} / \mathrm{dL}, \mathrm{AST}<40 \mathrm{U} / \mathrm{L}, \mathrm{ALT}<56 \mathrm{U} / \mathrm{L}$, and HbAlc levels of $5.7-6.4 \%(p<0.05)$. The higher prevalence of severe NAFLD was among 65 years and older, had high school education, obese, former smokers, did not meet physical activity guideline, eat poor quality diet, had high waist -to-hip ratio, $\mathrm{HDL}<40 \mathrm{mg} / \mathrm{dL}$, triglyceride $>200 \mathrm{mg} / \mathrm{dL}, \mathrm{CRP}>10 \mathrm{mg} / \mathrm{dL}$, AST $>40 \mathrm{U} / \mathrm{L}$, ALT $>56 \mathrm{U} / \mathrm{L}$, and HbAlc $>6.5 \%(p<0.05)$.

Table 3 and Figure 2 show the age-adjusted prevalence of NAFLD stage by race/ethnicity, gender and menopausal status with and without female hormone therapy. Of the 1,696 females, 510 were menopausal (out of these, 110 females used hormone therapy). Overall, the age adjusted prevalence of moderate NAFLD was highest among menopausal females who used 
TABLE 2 | Prevalence of NAFLD stages among male and female.

\begin{tabular}{|c|c|c|c|c|c|c|c|c|c|c|}
\hline & \multicolumn{5}{|c|}{ Male } & \multicolumn{5}{|c|}{ Female } \\
\hline & Overall & $\begin{array}{c}\text { No/mild } \\
(<260)\end{array}$ & $\begin{array}{l}\text { Moderate } \\
(260-290)\end{array}$ & $\begin{array}{l}\text { Severe } \\
(>290)\end{array}$ & $p$-value & Overall & $\begin{array}{c}\text { No/mild } \\
(<260)\end{array}$ & $\begin{array}{l}\text { Moderate } \\
(260-290)\end{array}$ & $\begin{array}{l}\text { Severe } \\
(>290)\end{array}$ & $p$-value \\
\hline Overall & $\begin{array}{c}1,596 \\
(48.2 \%)\end{array}$ & $745(47.5 \%)$ & 254 (16.0\%) & 597 (36.5\%) & & $\begin{array}{c}1,696 \\
(51.8 \%)\end{array}$ & $923(56.4 \%)$ & $285(16.2 \%)$ & $488(27.4 \%)$ & \\
\hline Race/ethnicity & & & & & $<0.0001$ & & & & & 0.059 \\
\hline Mexican American & $212(8.8)$ & $62(28.3)$ & 39 (17.6) & $111(54.1)$ & & 229 (8.1) & 95 (45.5) & $36(16.8)$ & $98(37.7)$ & \\
\hline Other Hispanic & $140(6.2)$ & $66(56.3)$ & $23(14.8)$ & $51(28.8)$ & & $146(5.9)$ & $81(56.4)$ & $21(12.7)$ & $44(30.9)$ & \\
\hline Non-Hispanic White & $588(65.7)$ & 259 (47.6) & 90 (15.3) & $239(37.1)$ & & $632(65.6)$ & $346(56.9)$ & $104(15.7)$ & $182(27.4)$ & \\
\hline Non-Hispanic Black & $348(9.5)$ & $208(60.7)$ & $50(15.3)$ & $90(24.0)$ & & 370 (10.3) & $216(61.6)$ & $65(16.7)$ & $89(21.6)$ & \\
\hline Other race & $308(9.8)$ & $150(45.6)$ & $52(21.1)$ & $106(33.3)$ & & $319(10.1)$ & $185(57.0)$ & $59(20.2)$ & 75 (22.9) & \\
\hline Age (years) & & & & & $<0.0001$ & & & & & $<0.0001$ \\
\hline 18-19 & $80(3.3)$ & $59(81.1)$ & $5(5.8)$ & $16(13.1)$ & & $85(3.2)$ & 71 (86.3) & $5(5.1)$ & $9(8.5)$ & \\
\hline 20-34 & $356(29.7)$ & $206(60.3)$ & $47(10.9)$ & $103(28.7)$ & & $386(25.1)$ & $273(71.8)$ & $50(12.1)$ & $63(16.1)$ & \\
\hline $35-49$ & $334(23.7)$ & $146(47.3)$ & $52(15.9)$ & $136(36.8)$ & & 394 (23.6) & $206(54.6)$ & $72(18.8)$ & $116(26.5)$ & \\
\hline $50-64$ & $447(26.8)$ & $172(34.7)$ & 85 (19.9) & $190(45.4)$ & & 475 (28.3) & $205(48.7)$ & $88(17.5)$ & $182(33.8)$ & \\
\hline 65+ & 379 (16.5) & 162 (38.5) & 65 (21.1) & $152(40.4)$ & & $356(19.8)$ & $168(45.4)$ & 70 (17.9) & $118(36.8)$ & \\
\hline Education & & & & & 0.068 & & & & & 0.039 \\
\hline Less than high school & $322(11.0)$ & $153(46.3)$ & $54(21.7)$ & $115(31.9)$ & & $254(8.8)$ & $133(55.3)$ & $41(17.3)$ & $80(27.5)$ & \\
\hline High school & $406(29.6)$ & $200(48.6)$ & $61(12.7)$ & $145(38.7)$ & & $415(26.8)$ & $221(52.6)$ & $59(15.2)$ & $135(32.2)$ & \\
\hline Some college & $484(29.0)$ & $215(45.7)$ & $70(12.8)$ & $199(41.5)$ & & $613(32.5)$ & $321(52.0)$ & $110(17.0)$ & $182(30.9)$ & \\
\hline At least college degree & $384(30.3)$ & $177(48.5)$ & $69(20.4)$ & $138(31.2)$ & & $414(31.9)$ & $248(64.6)$ & $75(15.7)$ & $91(19.7)$ & \\
\hline Language spoken at home & & & & & 0.522 & & & & & 0.182 \\
\hline English & 1,141 (82.2) & $556(48.2)$ & $176(16.4)$ & 409 (35.4) & & $1,217(83.6)$ & $688(57.8)$ & $200(15.7)$ & 329 (26.5) & \\
\hline Spanish & $106(3.5)$ & 34 (33.3) & $20(18.0)$ & $52(48.6)$ & & $113(3.5)$ & $49(44.7)$ & $20(16.8)$ & $44(38.5)$ & \\
\hline Both & $173(7.7)$ & $70(45.2)$ & $26(12.2)$ & $77(42.6)$ & & $184(7.1)$ & $85(49.8)$ & $27(16.2)$ & $72(34.0)$ & \\
\hline Other & $176(6.6)$ & $85(48.4)$ & $32(15.4)$ & $59(36.2)$ & & $182(5.9)$ & $101(52.7)$ & $38(21.6)$ & $43(25.7)$ & \\
\hline Federal income ratio (FIR) & & & & & 0.108 & & & & & 0.648 \\
\hline$<1$ & $281(10.6)$ & $157(58.8)$ & $29(10.2)$ & $95(31.0)$ & & $335(14.1)$ & $188(56.5)$ & $51(15.2)$ & $96(28.3)$ & \\
\hline $1-2$ & 430 (18.4) & $190(47.2)$ & $70(16.1)$ & $170(36.7)$ & & $460(20.7)$ & 245 (55.5) & 85 (19.5) & $130(25.0)$ & \\
\hline$>2$ & $885(71.1)$ & 398 (45.9) & $155(16.9)$ & $332(37.2)$ & & $901(65.2)$ & $490(56.7)$ & $149(15.3)$ & $262(28.0)$ & \\
\hline Waist-hip ratio & & & & & $<0.0001$ & & & & & $<0.0001$ \\
\hline Healthy & $990(64.6)$ & $593(62.2)$ & $160(14.7)$ & $237(23.1)$ & & $452(29.4)$ & $373(86.7)$ & $43(6.8)$ & $36(6.5)$ & \\
\hline $\begin{array}{l}\text { Risk for women ( } \geq 0.85 \%) \text { /risk } \\
\text { for men }(\geq 1.0 \%)\end{array}$ & $606(35.4)$ & $152(20.6)$ & $94(18.4)$ & $360(61.0)$ & & $1,244(70.6)$ & $550(43.8)$ & $242(20.0)$ & $452(36.1)$ & \\
\hline BMI & & & & & $<0.0001$ & & & & & $<0.0001$ \\
\hline Normal or healthy (<25) & $416(23.5)$ & $350(87.8)$ & $35(6.2)$ & $31(6.0)$ & & $518(32.2)$ & $443(89.4)$ & $48(7.4)$ & $27(3.2)$ & \\
\hline Overweight $(25-<30)$ & $586(35.2)$ & $280(50.8)$ & $130(23.8)$ & $176(25.4)$ & & $465(27.0)$ & $264(57.9)$ & $78(17.0)$ & $123(25.1)$ & \\
\hline
\end{tabular}


TABLE 2 | Continued

\begin{tabular}{|c|c|c|c|c|c|c|c|c|c|c|}
\hline & \multicolumn{5}{|c|}{ Male } & \multicolumn{5}{|c|}{ Female } \\
\hline & Overall & $\begin{array}{l}\text { No/mild } \\
(<260)\end{array}$ & $\begin{array}{l}\text { Moderate } \\
(260-290)\end{array}$ & $\begin{array}{l}\text { Severe } \\
(>290)\end{array}$ & $p$-value & Overall & $\begin{array}{l}\text { No/mild } \\
(<260)\end{array}$ & $\begin{array}{l}\text { Moderate } \\
(260-290)\end{array}$ & $\begin{array}{l}\text { Severe } \\
(>290)\end{array}$ & $p$-value \\
\hline Obese $(\geq 30)$ & $594(41.3)$ & $115(21.6)$ & 89 (15.0) & $390(63.3)$ & & $713(40.8)$ & $216(29.5)$ & $159(22.5)$ & $338(48.1)$ & \\
\hline Smoking status & & & & & 0.001 & & & & & 0.001 \\
\hline Current & $317(17.1)$ & $203(64.9)$ & $33(11.7)$ & $81(23.4)$ & & $233(14.2)$ & $130(54.8)$ & 37 (18.6) & $66(26.5)$ & \\
\hline Former & $466(31.1)$ & $168(38.4)$ & $89(19.3)$ & $209(42.3)$ & & $292(18.0)$ & $134(46.7)$ & $48(18.0)$ & $110(35.4)$ & \\
\hline Non-smoker & $813(51.7)$ & $374(47.2)$ & $132(15.5)$ & 307 (37.3) & & $1,171(67.8)$ & $659(59.4)$ & $200(15.1)$ & $312(25.5)$ & \\
\hline Physical activity & & & & & 0.024 & & & & & $<0.0001$ \\
\hline Inactive & 294 (14.3) & $113(36.7)$ & 45 (15.3) & $136(48.0)$ & & 439 (22.5) & 205 (48.2) & $88(19.0)$ & $146(32.9)$ & \\
\hline Does not meet guideline & 205 (12.6) & $88(38.1)$ & $28(17.2)$ & $89(44.7)$ & & $310(17.7)$ & $153(46.6)$ & $49(12.8)$ & $108(40.6)$ & \\
\hline Meets guidelines & 1,097 (73.1) & $544(51.2)$ & $181(16.0)$ & $372(32.8)$ & & $947(59.8)$ & $565(62.5)$ & $148(16.1)$ & $234(21.4)$ & \\
\hline Healthy eating index & & & & & 0.537 & & & & & $<0.0001$ \\
\hline Poor diet & $1,154(72.9)$ & $542(46.9)$ & $179(15.5)$ & $433(37.6)$ & & $1,150(70.0)$ & 615 (52.5) & $200(18.0)$ & $335(29.4)$ & \\
\hline Needs improvement & $404(25.0)$ & $185(47.7)$ & 68 (18.3) & $151(34.0)$ & & $483(26.8)$ & $275(64.7)$ & $72(11.7)$ & $136(23.6)$ & \\
\hline Good diet & $38(2.0)$ & $18(64.7)$ & $7(8.6)$ & $13(26.7)$ & & $63(3.1)$ & $33(73.2)$ & $13(12.2)$ & $17(14.5)$ & \\
\hline Serum cholesterol & & & & & 0.006 & & & & & 0.125 \\
\hline Good (<200 mg/dL) & $1,075(65.9)$ & $527(50.8)$ & $167(16.5)$ & $381(32.8)$ & & 1,062 (59.7) & $616(59.7)$ & $163(14.9)$ & $283(25.4)$ & \\
\hline Elevated (200-239 mg/dL) & $374(24.6)$ & $163(43.8)$ & $64(16.1)$ & $147(40.1)$ & & $451(29.4)$ & $221(50.7)$ & $83(16.7)$ & $147(32.6)$ & \\
\hline High ( $\geq 240$ mg/dL) & $147(9.5)$ & $55(34.1)$ & $23(12.7)$ & 69 (53.2) & & $183(10.9)$ & $86(53.7)$ & $39(21.7)$ & $58(24.6)$ & \\
\hline High-density lipoproteins & & & & & $<0.0001$ & & & & & $<0.0001$ \\
\hline Low (<40 mg/dL) & $410(23.9)$ & $118(30.5)$ & $69(15.4)$ & $223(54.1)$ & & $146(7.6)$ & $39(22.2)$ & $22(15.1)$ & $85(62.7)$ & \\
\hline Borderline risk (40-59 mg/dL) & $920(60.7)$ & $444(47.9)$ & $147(16.9)$ & $329(35.1)$ & & $865(48.8)$ & $409(48.7)$ & $154(16.9)$ & $302(34.4)$ & \\
\hline Healthy ( $\geq 60$ mg/dL) & 266 (15.5) & $183(71.9)$ & $38(13.6)$ & $45(14.6)$ & & $685(43.6)$ & $475(71.0)$ & $109(15.5)$ & $101(13.5)$ & \\
\hline Serum triglycerides & & & & & $<0.0001$ & & & & & $<0.0001$ \\
\hline Normal (<150 mg/dL) & $990(61.0)$ & $575(60.0)$ & $143(15.0)$ & $272(24.9)$ & & $1,246(73.5)$ & $795(66.3)$ & $196(14.8)$ & $255(18.9)$ & \\
\hline Borderline (150-199 mg/dL) & 255 (16.3) & 89 (33.5) & $56(21.3)$ & $110(45.2)$ & & $250(15.4)$ & $76(34.8)$ & $57(24.0)$ & $117(41.3)$ & \\
\hline High ( $\geq 200$ mg/dL) & 351 (22.6) & $81(23.7)$ & $55(14.9)$ & $215(61.4)$ & & $200(11.1)$ & $52(21.2)$ & $32(14.1)$ & $116(64.7)$ & \\
\hline High-sensitivity CRP & & & & & $<0.0001$ & & & & & $<0.0001$ \\
\hline Normal (0.1-<1mg/dL) & $522(33.1)$ & $331(65.8)$ & $72(13.1)$ & $119(21.1)$ & & $482(29.4)$ & $374(78.4)$ & $65(11.8)$ & $43(9.9)$ & \\
\hline $\begin{array}{l}\text { Mild inflammation }(1-<3 \\
\mathrm{mg} / \mathrm{dL})\end{array}$ & $635(40.8)$ & 265 (42.2) & $115(19.7)$ & $255(38.0)$ & & $536(31.8)$ & $294(57.4)$ & $102(19.6)$ & $140(23.0)$ & \\
\hline $\begin{array}{l}\text { Significant inflammation } \\
(3-<10 \mathrm{mg} / \mathrm{dL})\end{array}$ & $370(21.9)$ & $125(33.1)$ & $52(14.4)$ & $193(52.5)$ & & $514(29.6)$ & $203(40.9)$ & $92(16.4)$ & $219(42.8)$ & \\
\hline $\begin{array}{l}\text { High significant inflammation } \\
\text { ( } \geq 10 \mathrm{mg} / \mathrm{dL})\end{array}$ & $69(4.2)$ & $24(29.1)$ & $15(11.4)$ & $30(59.4)$ & & $164(9.1)$ & $52(33.1)$ & $26(17.5)$ & $86(49.4)$ & \\
\hline $\begin{array}{l}\text { Aspartate aminotransferase } \\
\text { (AST) }\end{array}$ & & & & & 0.452 & & & & & 0.012 \\
\hline Normal ( $\leq 40$ U/L) & $1,518(94.3)$ & $714(47.9)$ & 247 (16.3) & 557 (35.8) & & $1,647(97.7)$ & 903 (56.9) & 277 (16.2) & 467 (26.9) & \\
\hline
\end{tabular}




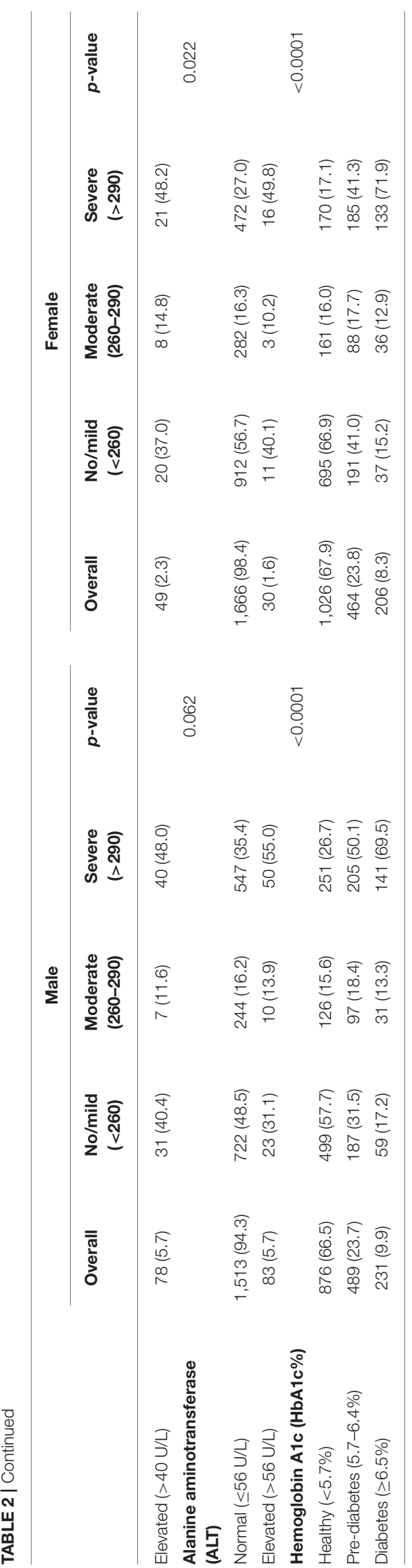

TABLE 3 | Age-adjusted prevalence of NAFLD stage by race/ethnicity and gender.

\begin{tabular}{|c|c|c|c|c|c|}
\hline Variable & $\begin{array}{c}\text { Male } \\
(n=1,596)\end{array}$ & $\begin{array}{l}\text { Female no } \\
\text { menopause } \\
(N=1,186)\end{array}$ & $\begin{array}{c}\text { Female } \\
\text { menopause } \\
\text { no hormone } \\
(N=400)\end{array}$ & $\begin{array}{c}\text { Female } \\
\text { menopause } \\
\text { plus } \\
\text { hormone } \\
(N=110)\end{array}$ & $p$-value \\
\hline Overall & & & & & $<0.001$ \\
\hline Normal/mild & 745 (48.12) & 689 (57.60) & $179(45.20)$ & 55 (23.44) & \\
\hline Moderate & 254 (15.86) & 193 (16.07) & 70 (24.72) & 22 (43.08) & \\
\hline Severe & 597 (36.01) & 304 (26.32) & 151 (30.08) & 33 (33.47) & \\
\hline $\begin{array}{l}\text { Mexican } \\
\text { American }\end{array}$ & & & & & $<0.001$ \\
\hline Normal/mild & 62 (26.45) & $80(46.10)$ & $12(57.30)$ & 3 (49.31) & \\
\hline Moderate & 39 (17.70) & $26(16.97)$ & $9(15.57)$ & $1(7.60)$ & \\
\hline Severe & $111(55.83)$ & 67 (36.92) & $25(27.14)$ & $6(43.09)$ & \\
\hline $\begin{array}{l}\text { Other } \\
\text { Hispanics }\end{array}$ & & & & & 0.9945 \\
\hline Normal/mild & 66 (56.04) & 53 (58.11) & 21 (54.90) & $7(49.57)$ & \\
\hline Moderate & $23(14.49)$ & $11(12.43)$ & $8(14.66)$ & $2(16.40)$ & \\
\hline Severe & $51(29.45)$ & 27 (29.45) & $12(30.45)$ & 5 (34.03) & \\
\hline Blacks & & & & & 0.4346 \\
\hline Normal/mild & 208 (60.19) & $164(60.38)$ & $41(60.67)$ & $11(40.59)$ & \\
\hline Moderate & $50(15.37)$ & 47 (17.55) & 14 (15.32) & $4(13.48)$ & \\
\hline Severe & $90(24.43)$ & $53(22.07)$ & 34 (24.03) & $2(45.93)$ & \\
\hline Whites & & & & & $<0.001$ \\
\hline Normal/mild & 259 (49.02) & 253 (59.36) & 69 (48.28) & $24(21.46)$ & \\
\hline Moderate & 90 (14.95) & 68 (15.19) & 24 (16.95) & $12(47.91)$ & \\
\hline Severe & 239 (36.01) & $114(25.45)$ & $51(34.77)$ & $17(30.62)$ & \\
\hline Others & & & & & 0.0083 \\
\hline Normal/mild & $150(47.37)$ & $139(57.40)$ & 36 (29.23) & $10(47.37)$ & \\
\hline Moderate & 52 (19.73) & 41 (24.59) & 15 (41.49) & $3(19.89)$ & \\
\hline Severe & 106 (32.89) & 43 (18.00) & 29 (29.28) & 3 (32.89) & \\
\hline
\end{tabular}

hormone therapy (43.1\%) followed by menopausal females with no hormone therapy $(24.7 \%)$, then non-menopausal females $(16.1 \%)$ and males $(15.9 \%)(p<0.05)$. Overall, the age adjusted prevalence of severe NAFLD was highest among males (36\%), followed by menopausal females who used hormone therapy (33.5\%), followed by menopausal females with no hormone therapy (30.1\%), then non-menopausal females (26.3\%) ( $p$ $<0.05)$. Among Mexican Americans, significantly higher prevalence of severe NAFLD was found in males (55.8\%), followed by menopausal females with hormone therapy (43.1\%). Among other Hispanics and Blacks, severe NAFLD was higher among menopausal females who used hormone therapy (34.0 and $45.9 \%$, respectively). Among Whites and other race/ethnicity groups, higher prevalence of severe NAFLD was found among males (36.0 and 32.9\%, respectively) relative to females (Table 3 ).

\section{Factors Associated With NAFLD Stages}

In the multinomial adjusted model (Table 4), race/ethnicity was significantly associated with NAFLD stage. Mexican Americans were two times more likely to have moderate NAFLD [adjusted 


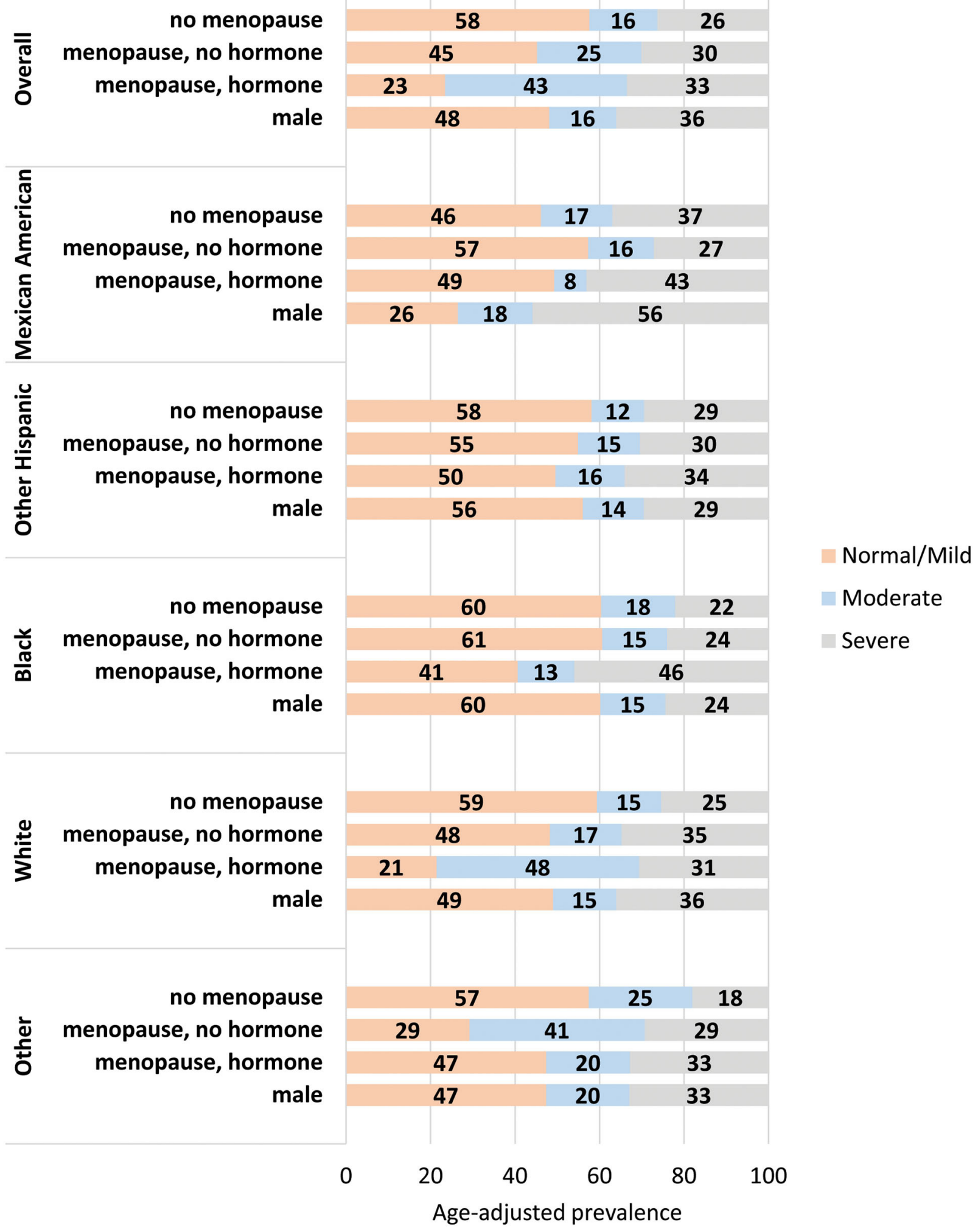


TABLE 4 | Adjusted odds ratio (OR) and 95\% confidence interval (Cl) for the relationship between NAFLD and race/ethnicity (reference $=$ no/mild NAFLD).

\begin{tabular}{lccccc}
\hline $\begin{array}{l}\text { Reference }= \\
\text { no/mild NAFLD }\end{array}$ & \multicolumn{2}{c}{ Moderate (260-290) } & & \multicolumn{2}{c}{ Severe (>290) } \\
\cline { 2 - 3 } \cline { 5 - 6 } & $\begin{array}{c}\text { AOR [95\% } \\
\mathrm{Cl}]\end{array}$ & $p$-value & & $\begin{array}{c}\text { AOR [95\% } \\
\mathrm{Cl}]\end{array}$ & $p$-value
\end{tabular}

\section{Race/Ethnicity}

\section{Mexican-}

American vs.

non-Hispanic

White

Other Hispanic

vs. non-Hispanic

White

Non-Hispanic

Black vs.

non-Hispanic

White

\section{Other race vs.}

non-Hispanic

White

\section{Age (years)}

18-19 vs. 20-34

35-49 vs. 20-34

50-64 vs. 20-34

65+ vs. 20-34

Education

Less than high

school vs. high

school

Some college

vs. high school

At least college

degree vs. high

school

\section{Language spoken at home}

\begin{tabular}{|c|c|c|c|c|}
\hline $\begin{array}{l}\text { Spanish vs. } \\
\text { English }\end{array}$ & $0.8[0.5-1.4]$ & 0.4419 & $0.8[0.5-1.4]$ & 0.4560 \\
\hline Both vs. English & $0.8[0.5-1.4]$ & 0.4874 & $1.1[0.6-2.0]$ & 0.8350 \\
\hline Other vs. English & $1.2[0.6-2.5]$ & 0.5989 & $1.5[0.8-3.0]$ & 0.2312 \\
\hline \multicolumn{5}{|c|}{ Federal income ratio (FIR) } \\
\hline$<1$ vs. $>2$ & $0.8[0.6-1.1]$ & 0.2188 & $0.8[0.6-1.2]$ & 0.2640 \\
\hline $1-2$ vs. $>2$ & $1.1[0.7-1.8]$ & 0.6418 & $0.9[0.6-1.2]$ & 0.3799 \\
\hline \multicolumn{5}{|l|}{ Waist-hip ratio } \\
\hline $\begin{array}{l}\text { Risk for women } \\
(\geq 0.85) / \text { risk for } \\
\text { men }(\geq 1.0) \text { vs. } \\
\text { healthy }\end{array}$ & $1.7[1.1-2.8]$ & 0.0249 & $1.8[1.2-2.7]$ & 0.0041 \\
\hline \multicolumn{5}{|l|}{ BMI } \\
\hline $\begin{array}{l}\text { Overweight } \\
(25-<30) \text { vs. } \\
\text { normal }(<25)\end{array}$ & $3.9[2.4-6.6]$ & $<0.0001$ & $\begin{array}{c}7.1 \\
{[3.9-13.1]}\end{array}$ & $<0.0001$ \\
\hline $\begin{array}{l}\text { Obese }(\geq 30) \text { vs. } \\
\text { normal }(<25)\end{array}$ & $\begin{array}{c}6.5 \\
{[3.7-11.6]}\end{array}$ & $<0.0001$ & $\begin{array}{c}24.8 \\
{[11.9-51.9]}\end{array}$ & $<0.0001$ \\
\hline \multicolumn{5}{|l|}{ Smoking status } \\
\hline Current vs. never & $0.9[0.6-1.4]$ & 0.5794 & $0.7[0.5-1.1]$ & 0.1166 \\
\hline Former vs. never & $1.2[0.9-1.6]$ & 0.3316 & $1.2[0.9-1.7]$ & 0.2279 \\
\hline
\end{tabular}

(Continued)
TABLE 4 | Continued

\begin{tabular}{lccccc}
\hline $\begin{array}{l}\text { Reference }= \\
\text { no/mild NAFLD }\end{array}$ & \multicolumn{2}{c}{ Moderate (260-290) } & & Severe $(>290)$ \\
\cline { 2 - 3 } \cline { 5 - 6 } & $\begin{array}{c}\text { AOR [95\% } \\
\text { Cl] }\end{array}$ & $p$-value & & $\begin{array}{c}\text { AOR [95\% } \\
\text { Cl] }\end{array}$ & $p$-value
\end{tabular}

\section{Alcohol use}

Current vs. never

Former vs. never

1.4 [0.9-2.3]

0.1269

$0.7[0.4-1.2]$

0.1888

Physical activity

$1.4[0.9-2.3]$

0.1717

$0.6[0.3-1.1]$

0.1136

Inactive vs.

1.0

Does not meet $\quad 0.9[0.5-1.6] \quad 0.6670 \quad 1.4[0.9-2.2] \quad 0.1557$

guidelines vs.

meets guidelines

Healthy eating index

$\begin{array}{lllll}\text { Poor diet vs. } & 1.6[0.7-3.9] & 0.2610 & 0.9[0.2-3.9] & 0.9219 \\ \text { good diet } & & & \end{array}$

$\begin{array}{lllll}\text { Needs } & 1.2[0.5-3.3] & 0.6525 & 0.9[0.2-3.4] & 0.8771\end{array}$

improvement vs.

good diet

Serum cholesterol

Elevated

0.8 [0.5-1.2]

$0.9[0.7-1.3]$

0.7105

(200-239

$\mathrm{mg} / \mathrm{dL}) \mathrm{vs}$. good

(<200 mg/dL)

High ( $\geq 240$

$\mathrm{mg} / \mathrm{dL})$ vs. good

( $<200 \mathrm{mg} / \mathrm{dL}$ )

\section{High-density lipoproteins}

$\begin{array}{lllll}\text { Low }(<40 & 1.2[0.6-2.7] & 0.5843 & \mathbf{2 . 7}[\mathbf{1 . 6 - 4 . 7}] & \mathbf{0 . 0 0 0 3} \\ \mathrm{mg} / \mathrm{dL}) \text { vs. } & & & \\ \text { healthy }(\geq 60 & & & \\ \mathrm{mg} / \mathrm{dL}) & & & \\ \begin{array}{l}\text { Borderline risk } \\ \text { (40-59 mg/dL) }\end{array} & 1.2[0.8-1.7] & 0.4582 & \mathbf{1 . 8}[\mathbf{1 . 2 - 2 . 6 ]} & \mathbf{0 . 0 0 3 2} \\ \text { vs. healthy }(\geq 60 & & & & \\ \mathrm{mg} / \mathrm{dL})\end{array}$

\section{Serum triglycerides}

$\begin{array}{lllll}\text { Borderline } & 1.8[1.3-2.4] & 0.0009 & 1.7[1.0-2.8] & 0.0375\end{array}$

(150-199

$\mathrm{mg} / \mathrm{dL}) \mathrm{vs}$.

normal $(<150$

$\mathrm{mg} / \mathrm{dL})$

High $(\geq 200 \quad 1.7[1.3-2.4] \quad 0.0007 \quad 2.9[1.9-4.4] \quad<0.0001$

$\mathrm{mg} / \mathrm{dL}) \mathrm{vs}$.

normal $(<150$

$\mathrm{mg} / \mathrm{dL}$ )

High-sensitivity CRP

$\begin{array}{lllll}\begin{array}{l}\text { Mild } \\ \text { inflammation }\end{array} & 1.4[0.9-2.2] & 0.1638 & 1.2[0.7-2.0] & 0.4566 \\ \begin{array}{l}(1-3 \mathrm{mg} / \mathrm{dL}) \text { vs. } \\ \text { normal }(<1\end{array} & & & & \\ \mathrm{mg} / \mathrm{dL}) & & & & \\ \text { Significant } & 1.1[0.7-1.7] & 0.7856 & 1.4[0.8-2.5] & 0.2684 \\ \text { inflammation } & & & & \\ (3-10 \mathrm{mg} / \mathrm{dL}) \text { vs. } & & & \\ \text { normal }(<1 & & & & \\ \mathrm{mg} / \mathrm{dL})\end{array}$

(Continued) 
TABLE 4 | Continued

\begin{tabular}{|c|c|c|c|c|}
\hline \multirow{2}{*}{$\begin{array}{l}\text { Reference = } \\
\text { no/mild NAFLD }\end{array}$} & \multicolumn{2}{|c|}{ Moderate (260-290) } & \multicolumn{2}{|c|}{ Severe $(>290)$} \\
\hline & $\begin{array}{c}\text { AOR }[95 \% \\
\mathrm{Cl}]\end{array}$ & $p$-value & $\begin{array}{c}\text { AOR }[95 \% \\
\mathrm{Cl}]\end{array}$ & $p$-value \\
\hline $\begin{array}{l}\text { High significant } \\
\text { inflammation } \\
\text { ( } \geq 10 \mathrm{mg} / \mathrm{dL})\end{array}$ & $1.3[0.8-2.0]$ & 0.2317 & $1.8[0.8-4.0]$ & 0.1597 \\
\hline \multicolumn{5}{|c|}{ Aspartate aminotransferase (AST) } \\
\hline $\begin{array}{l}\text { Elevated (>40 } \\
\text { U/L) vs. normal } \\
\text { ( } \leq 40 \text { U/L) }\end{array}$ & $1.4[0.3-6.3]$ & 0.6815 & $1.9[0.7-5.0]$ & 0.2018 \\
\hline \multicolumn{5}{|c|}{ Alanine aminotransferase (ALT) } \\
\hline $\begin{array}{l}\text { Elevated (>56 } \\
\text { U/L) vs. normal } \\
\text { ( } \leq 56 \text { U/L) }\end{array}$ & $1.3[0.3-4.6]$ & 0.7310 & $1.9[0.6-6.2]$ & 0.2611 \\
\hline \multicolumn{5}{|c|}{ Hemoglobin A1c (HbA1c) } \\
\hline $\begin{array}{l}\text { Pre-diabetes vs. } \\
\text { healthy }(<5.7 \%)\end{array}$ & $1.3[0.9-1.9]$ & 0.2221 & $2.4[1.7-3.6]$ & $<0.0001$ \\
\hline $\begin{array}{l}\text { Diabetes } \\
(\geq 6.5 \%) \text { vs. } \\
\text { healthy }(<5.7 \%)\end{array}$ & $1.6[0.9-2.8]$ & 0.1469 & $5.0[2.6-9.3]$ & $<0.0001$ \\
\hline
\end{tabular}

Bold $=$ statistically significant at $p<0.05$.

TABLE 5 | Adjusted odds ratio (AOR) and 95\% confidence interval (Cl) of the association between NAFLD stage and gender.

\begin{tabular}{lcc}
\hline Reference $=$ normal/mild NAFLD & \multicolumn{2}{c}{ NAFLD } \\
\cline { 2 - 3 } & $\begin{array}{c}\text { Moderate } \\
\text { AOR [95\% Cl] }\end{array}$ & $\begin{array}{c}\text { Severe } \\
\text { AOR [95\% Cl] }\end{array}$ \\
\hline No menopause & Ref & Ref \\
Menopause, no hormone & $0.9[0.6-1.3]$ & $1.0[0.5-1.9]$ \\
Menopause, yes hormone & $1.1[0.4-2.9]$ & $1.1[0.4-2.8]$ \\
Male & $1.4[0.9-2.1]$ & $\mathbf{2 . 1}[\mathbf{1 . 5 - 2 . 9}]$
\end{tabular}

Adjusted for demographic variables (age, race/ethnicity, education, language spoken, and poverty), physical activity status, smoking status, diet quality (healthy eating index), body composition (waist-to-hip ratio and body mass index), and laboratory values [cholesterol, $H D L$, triglyceride, glucose, hemoglobin A1c $(H b A 1 C)$, highly-sensitive C-reactive protein (hSCRP), AST, and ALT].

Bold $=$ statistically significant at $p<0.05$.

odds ratio $(\mathrm{AOR})=1.9,95 \%$ confidence interval $(\mathrm{CI})=1.1-$ $3.4, p<0.05]$ and were more than twice as likely to have severe NAFLD $(\mathrm{AOR}=2.4,95 \% \mathrm{CI}=1.4-4.2, p<0.05)$ relative to the White population. In addition, the Black population was significantly less likely to have severe NAFLD relative to White population $(\mathrm{AOR}=0.5,95 \% \mathrm{CI}=0.4-0.7, p<0.05)$.

Relative to the age group of 20-34 years of age, the 50-64 years and 65+ years old groups were about two times more likely to have moderate NAFLD; the 50-64 years old group was also two times more likely to have severe NAFLD $(p<0.05)$. Participants in the high-risk group of the waist-to-hip ratio were about two times more likely to have moderate NAFLD and to have severe NAFLD $(p<0.05)$ relative to those in the normal waist-to-hip ratio group. Relative to the group with normal BMI, overweight participants were 3.9 times more likely to have moderate NAFLD and about seven times more likely to have severe NAFLD $(<0.05)$. This relationship was stronger among the obese population for both moderate NAFLD (six times higher odds) and severe NAFLD (25 times higher odds) $(p<0.05)$.

Participants with borderline and low HDL levels had higher odds of developing severe NAFLD than those with a normal level of HDL $(p<0.05)$. Participants with borderline and high levels of triglycerides had higher odds than those with normal levels to have moderate and severe NAFLD $(p<0.05)$. Subjects with high $\mathrm{HbA1c}$ in both groups of pre-diabetes and diabetes had higher odds of severe NAFLD relative to the normal group $(p<$ $0.05)$. Patients with pre-diabetes had twice the odds of developing severe NAFLD $(p<0.05)$ and patients with diabetes had 5 times higher odds of displaying severe NAFLD relative to the normal group (Table 4).

Table 5 and Figure 3 shows that after adjustment for the other independent variables, males had two times higher odds of severe NAFLD relative to non-menopausal females $(\mathrm{AOR}=2.1,95 \% \mathrm{CI}$ $=1.5-2.9, p<0.05)$. There was no difference in the likelihood of having moderate or severe NAFLD in the menopausal females with or without hormone therapy relative to non-menopausal females $(p>0.05)$.

\section{Gender Is an Effect Modifier of the Association Between Race/Ethnicity and NAFLD Stages}

In the stratified analysis (Table 6), after adjusting for the other independent variables, Mexican Americans had more than four times higher odds of moderate and severe NAFLD relative to Whites only among males $(p<0.05)$ and similar odds as Whites among females $(p>0.05)$. Whereas, non-Hispanic Blacks had lower odds of severe NAFLD relative to Whites among both males and females, after adjusting for other independent variables $(p<0.05)$.

\section{Factors Associated With Moderate NAFLD Stages Among Males and Females}

In the stratified multinomial adjusted model (Table 6), NAFLD was significantly associated with old age, a high waist-to-hip ratio, a high BMI, borderline/high triglyceride level, and pre-diabetes and diabetes diagnosis by HbA1c in both genders $(p<0.05)$. While age was not associated with moderate NAFLD in females $(p>0.05)$, males aged 50 years and older had over two-fold chances of developing moderate NAFLD relative to those of $20-$ 34 years of age $(p<0.05)$. Waist-to-hip ratio was not associated with moderate NAFLD among males $(p>0.05)$, but females with high waist-to-hip ratio were about three times more likely to have moderate NAFLD relative to females with a normal waist-tohip ratio $(p<0.05)$. Those who were overweight or obese were more likely than the normal group to have moderate NAFLD among both males and females $(p<0.05)$. While borderline triglyceride level was associated with moderate NAFLD among only females, high triglyceride level was associated with moderate NAFLD among males only $(p<0.05)$. High AST and ALT levels 


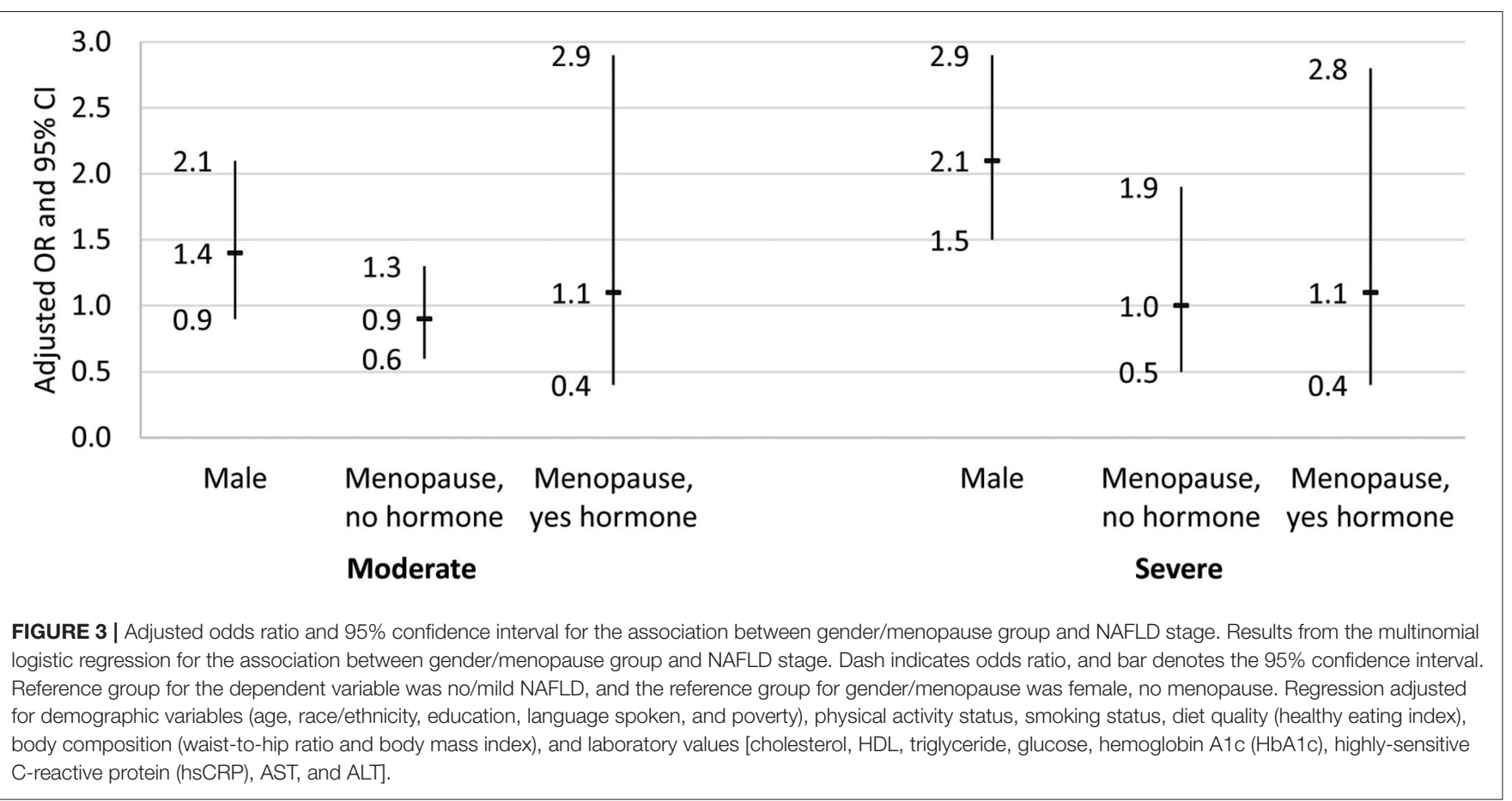

as well as the level of HbA1c were associated with moderate NAFLD only among females $(p<0.05)$ and not among males $(p>0.05$; Table 6).

Table 7 shows that among males, Mexican Americans had more than 4 times higher odds of moderate NAFLD relative to Whites $(\mathrm{AOR}=4.5[95 \% \mathrm{CI}=1.7-11.7], p<0.05)$. There was no association between race/ethnicity and moderate NAFLD among females with and without menopause $(p>0.05)$.

\section{Factors Associated With Severe NAFLD Stages Among Males and Females}

Severe NAFLD was associated with 50-64 years of age, high waist-to-hip ratio, high BMI, high levels of triglycerides, and prediabetes and diabetes status among both males and females $(p$ $<0.05$; Table 6). While females aged 65 years and older had more than two times higher odds of severe NAFLD $(p<0.05)$, this relation was not statistically significant among males $(p>$ 0.05). For males, current smokers had lower odds of having severe NAFLD than those who never smoked $(p>0.05)$; this relationship was not observed in females $(p>0.05)$. While physically inactive males had about twice the odds to develop severe NAFLD relative to physically active males $(p<0.05)$, physical inactivity did not play a significant role in females $(p>$ 0.05 ). While females with low HDL levels had about four times higher odds of severe NAFLD relative to females with normal HDL $(p<0.05)$, this association was not statistically significant among males $(p>0.05)$. In females, both borderline and high levels of triglycerides increased the odds of severe NAFLD, but in males only high levels increased the odds. In addition, females with high level of AST level had about five times higher odds of severe NAFLD relative to females with normal AST level $(p<$
0.05), but this association was not statistically significant among males $(p>0.05)$. In males, on the other hand, a high level of ALT was associated with a three times higher odds of severe NAFLD relative to males with a normal ALT level $(p<0.05)$; this association was not significant in females ( $p>0.05$; Table 6).

Table 7 shows that among males, Mexican Americans had five times higher odds of severe NAFLD relative to Whites (AOR $=5.0[95 \% \mathrm{CI}=2.1-12.0], p<0.05$ ). Blacks had lower odds of severe NAFLD relative to Whites among males and nonmenopausal females (AOR $=0.5[95 \% \mathrm{CI}=0.3-0.9], p<0.05$ and $\mathrm{AOR}=0.5[95 \% \mathrm{CI}=0.3-0.9], p<0.05)$.

\section{DISCUSSION}

The current study analyzed data from the NHANES 20172018 database and examined the association of gender with racial/ethnic differences with respect to NAFLD stages in the US population (normal/mild, moderate, and severe), as estimated by transient elastography. More than half of participants had no/mild NAFLD, with about one third having severe NAFLD. The findings from our study are consistent with other studies showing that $\sim 1$ in 3 subjects had NAFLD (12) with CAP> $290 \mathrm{~dB} / \mathrm{m}$. Importantly, Mexican Americans had the highest prevalence of severe NAFLD compared to non-Hispanic Whites, while other Hispanics had a lower prevalence than Whites. Among all racial/ethnic groups, blacks had the lowest prevalence of NAFLD. These findings are consistent with other studies showing that Hispanics have a higher prevalence of NAFLD and blacks a lower prevalence of NAFLD compared to non-Hispanic Whites $(2,17)$. 
TABLE 6 | Adjusted odds ratio (AOR) and 95\% confidence interval (Cl) for the relationship between NAFLD and race/ethnicity by gender (reference = no/mild NAFLD).

\begin{tabular}{|c|c|c|c|c|c|c|c|c|}
\hline \multirow[t]{3}{*}{ Reference $=$ no $/$ mild NAFLD } & \multicolumn{4}{|c|}{ Male } & \multicolumn{4}{|c|}{ Female } \\
\hline & \multicolumn{2}{|c|}{ Moderate (260-290) } & \multicolumn{2}{|c|}{ Severe $(>290)$} & \multicolumn{2}{|c|}{ Moderate (260-290) } & \multicolumn{2}{|c|}{ Severe $(>290)$} \\
\hline & AOR $[95 \% \mathrm{Cl}]$ & $p$-value & AOR $[95 \% \mathrm{Cl}]$ & $p$-value & AOR $[95 \% \mathrm{Cl}]$ & $p$-value & AOR $[95 \% \mathrm{Cl}]$ & $p$-value \\
\hline \multicolumn{9}{|l|}{ Race/Ethnicity } \\
\hline Mexican Americans vs. non-Hispanic White & $4.5[1.7-11.7]$ & 0.0020 & $5.0[2.1-12.0]$ & 0.0003 & $0.9[0.4-2.3]$ & 0.8628 & $1.8[0.6-5.8]$ & 0.3296 \\
\hline Other Hispanic vs. non-Hispanic White & $1.2[0.5-2.6]$ & 0.7152 & $0.7[0.3-2.2]$ & 0.5927 & $0.5[0.2-1.6]$ & 0.2700 & $0.9[0.3-2.9]$ & 0.9131 \\
\hline Non-Hispanic Black vs. non-Hispanic White & $1.0[0.5-1.9]$ & 0.9972 & $0.5[0.3-0.9]$ & 0.0212 & $0.7[0.5-1.1]$ & 0.1076 & $0.5[0.3-0.9]$ & 0.0101 \\
\hline Other race vs. non-Hispanic White & $2.3[0.9-5.9]$ & 0.0788 & $1.3[0.7-2.6]$ & 0.3878 & $1.2[0.6-2.5]$ & 0.6419 & $0.8[0.4-1.9]$ & 0.6667 \\
\hline \multicolumn{9}{|l|}{ Age (years) } \\
\hline $18-19$ vs. $20-34$ & $0.6[0.2-2.0]$ & 0.3815 & $0.6[0.3-1.3]$ & 0.2346 & $0.6[0.1-2.7]$ & 0.5121 & $0.6[0.2-2.2]$ & 0.4404 \\
\hline $35-49$ vs. $20-34$ & $1.3[0.6-2.9]$ & 0.5466 & $1.1[0.6-2.0]$ & 0.7388 & $1.6[0.8-3.2]$ & 0.2128 & $1.6[0.7-3.5]$ & 0.2865 \\
\hline 50-64 vs. $20-34$ & $2.8[1.5-5.6]$ & 0.0023 & $2.3[1.2-4.3]$ & 0.0111 & $1.9[0.9-3.8]$ & 0.0747 & $2.4[1.4-4.0]$ & 0.0014 \\
\hline $65+$ vs. 20-34 & $2.4[1.1-5.1]$ & 0.0202 & $1.4[0.7-2.8]$ & 0.3110 & $1.7[0.7-4.1]$ & 0.2375 & $2.2[1.1-4.7]$ & 0.0324 \\
\hline \multicolumn{9}{|l|}{ Education } \\
\hline Less than high school vs. high school & $2.2[1.1-4.2]$ & 0.0196 & $0.8[0.5-1.4]$ & 0.4710 & $1.0[0.6-1.7]$ & 0.8996 & $0.6[0.2-1.5]$ & 0.2680 \\
\hline Some college vs. high school & $0.9[0.5-1.5]$ & 0.6436 & $1.0[0.5-2.0]$ & 0.9845 & $1.5[0.9-2.8]$ & 0.1479 & $1.4[0.8-2.4]$ & 0.2869 \\
\hline At least college degree vs. high school & $1.3[0.6-2.7]$ & 0.5380 & $0.8[0.5-1.3]$ & 0.3531 & $1.4[0.6-3.0]$ & 0.4505 & $1.0[0.4-2.6]$ & 0.9502 \\
\hline \multicolumn{9}{|l|}{ Language spoken at home } \\
\hline Spanish vs. English & $0.4[0.1-1.1]$ & 0.0634 & $0.4[0.1-1.4]$ & 0.1506 & $2.1[0.6-7.6]$ & 0.2758 & $1.1[0.2-5.6]$ & 0.8652 \\
\hline Both vs. English & $0.3[0.1-1.0]$ & 0.0546 & $0.6[0.2-2.2]$ & 0.4489 & $2.1[0.8-5.6]$ & 0.1429 & $1.3[0.4-4.2]$ & 0.6568 \\
\hline Other vs. English & $0.8[0.4-1.6]$ & 0.4946 & $1.7[0.6-4.4]$ & 0.3139 & $1.8[0.7-4.6]$ & 0.2155 & $1.7[0.8-3.7]$ & 0.1983 \\
\hline \multicolumn{9}{|l|}{ Federal income ratio (FIR) } \\
\hline$<1$ vs. $>2$ & $0.7[0.3-1.4]$ & 0.2760 & $0.9[0.5-1.8]$ & 0.8686 & $0.9[0.5-1.6]$ & 0.6853 & $0.8[0.5-1.4]$ & 0.4980 \\
\hline $1-2$ vs. $>2$ & $1.2[0.8-1.7]$ & 0.4381 & $1.3[0.7-2.4]$ & 0.3633 & $1.1[0.6-2.0]$ & 0.8161 & $0.7[0.4-1.1]$ & 0.0815 \\
\hline \multicolumn{9}{|l|}{ Waist-hip ratio } \\
\hline $\begin{array}{l}\text { Risk for women }(\geq 0.85) / \text { risk for men }(\geq 1.0) \text { vs. } \\
\text { healthy }\end{array}$ & $1.6[0.8-2.9]$ & 0.1518 & $1.9[1.2-3.0]$ & 0.0036 & $3.0[1.3-6.8]$ & 0.0073 & $2.9[1.4-6.0]$ & 0.0042 \\
\hline \multicolumn{9}{|l|}{ BMI } \\
\hline Overweight $(25-<30)$ vs. normal $(<25)$ & $5.3[2.9-9.6]$ & $<0.0001$ & $5.9[3.2-11.2]$ & $<0.0001$ & $2.5[1.3-4.8]$ & 0.0053 & $8.1[4.3-15.3]$ & $<0.0001$ \\
\hline Obese $(\geq 30)$ vs. normal $(<25)$ & $6.9[3.3-14.6]$ & $<0.0001$ & $\begin{array}{c}30.6 \\
{[14.1-66.3]}\end{array}$ & $<0.0001$ & $6.4[3.4-12.0]$ & $<0.0001$ & $21.0[7.7-57.6]$ & $<0.0001$ \\
\hline \multicolumn{9}{|l|}{ Smoking status } \\
\hline Current vs. never & $0.5[0.2-1.4]$ & 0.1854 & $0.5[0.2-0.9]$ & 0.0209 & $1.2[0.8-1.8]$ & 0.4637 & $1.0[0.6-1.6]$ & 0.9229 \\
\hline Former vs. never & $1.0[0.6-1.8]$ & 0.8638 & $1.0[0.7-1.6]$ & 0.8662 & $1.0[0.6-1.8]$ & 0.9425 & $1.0[0.7-1.6]$ & 0.9411 \\
\hline \multicolumn{9}{|l|}{ Alcohol use } \\
\hline Current vs. never & $1.2[0.7-2.4]$ & 0.5136 & $0.6[0.3-1.3]$ & 0.2230 & $1.4[0.8-2.4]$ & 0.2807 & $0.7[0.3-1.5]$ & 0.3233 \\
\hline Former vs. never & $1.1[0.4-2.9]$ & 0.8811 & $0.4[0.2-0.9]$ & 0.0278 & $1.4[0.6-3.1]$ & 0.4336 & $0.7[0.3-1.6]$ & 0.4653 \\
\hline
\end{tabular}


TABLE 6 | Continued

\begin{tabular}{|c|c|c|c|c|c|c|c|c|}
\hline \multirow[t]{3}{*}{ Reference $=$ no/mild NAFLD } & \multicolumn{4}{|c|}{ Male } & \multicolumn{4}{|c|}{ Female } \\
\hline & \multicolumn{2}{|c|}{ Moderate (260-290) } & \multicolumn{2}{|c|}{ Severe $(>290)$} & \multicolumn{2}{|c|}{ Moderate (260-290) } & \multicolumn{2}{|c|}{ Severe $(>290)$} \\
\hline & AOR $[95 \% \mathrm{Cl}]$ & $p$-value & AOR $[95 \% \mathrm{Cl}]$ & $p$-value & AOR $[95 \% \mathrm{Cl}]$ & $p$-value & AOR $[95 \% \mathrm{Cl}]$ & $p$-value \\
\hline \multicolumn{9}{|l|}{ Physical activity } \\
\hline Inactive vs. meets guidelines & $1.1[0.5-2.2]$ & 0.7924 & $1.9[1.1-3.4]$ & 0.0258 & $1.0[0.6-1.6]$ & 0.9031 & $1.1[0.6-2.1]$ & 0.7934 \\
\hline Does not meet guidelines vs. meets guidelines & $1.0[0.5-2.1]$ & 0.9900 & $1.3[0.7-2.3]$ & 0.3704 & $0.8[0.4-1.5]$ & 0.5128 & $1.8[0.9-3.6]$ & 0.0707 \\
\hline \multicolumn{9}{|l|}{ Healthy eating index } \\
\hline Poor diet vs. good diet & $2.6[0.6-10.8]$ & 0.1841 & $0.9[0.1-7.7]$ & 0.9529 & $1.2[0.3-3.9]$ & 0.8100 & $0.9[0.3-3.0]$ & 0.9028 \\
\hline Needs improvement vs. good diet & $2.5[0.6-9.7]$ & 0.1947 & $1.2[0.2-9.0]$ & 0.8682 & $0.7[0.2-2.1]$ & 0.5085 & $0.7[0.3-1.9]$ & 0.4773 \\
\hline \multicolumn{9}{|l|}{ Serum cholesterol } \\
\hline Elevated (200-239 mg/dL) vs. good (<200 mg/dL) & $0.6[0.4-0.9]$ & 0.0243 & $0.6[0.3-1.1]$ & 0.1125 & $1.1[0.7-1.6]$ & 0.7665 & $1.2[0.8-2.0]$ & 0.3699 \\
\hline High ( $\geq 240$ mg/dL) vs. good (<200 mg/dL) & $0.5[0.2-1.2]$ & 0.1320 & $0.9[0.4-1.9]$ & 0.8289 & $1.1[0.6-1.8]$ & 0.8074 & $0.6[0.3-1.1]$ & 0.0947 \\
\hline \multicolumn{9}{|l|}{ High-density lipoproteins } \\
\hline Low (<40 mg/dL) vs. healthy ( $\geq 60 \mathrm{mg} / \mathrm{dL}$ ) & $1.0[0.4-2.6]$ & 0.9782 & $1.8[0.8-3.8]$ & 0.1262 & $1.5[0.3-6.8]$ & 0.6300 & $3.7[1.4-9.5]$ & 0.0067 \\
\hline $\begin{array}{l}\text { Borderline risk (40-59 mg/dL) vs. healthy ( } \geq 60 \\
\mathrm{mg} / \mathrm{dL} \text { ) }\end{array}$ & $1.2[0.7-2.1]$ & 0.5357 & $1.6[0.8-3.3]$ & 0.1722 & $0.9[0.6-1.5]$ & 0.7587 & $1.4[0.9-2.2]$ & 0.1465 \\
\hline \multicolumn{9}{|l|}{ Serum triglycerides } \\
\hline $\begin{array}{l}\text { Borderline (150-199 mg/dL) vs. normal (<150 } \\
\mathrm{mg} / \mathrm{dL})\end{array}$ & $1.9[1.0-3.5]$ & 0.0594 & $1.7[0.7-4.0]$ & 0.2692 & $1.9[1.2-2.8]$ & 0.0035 & $1.9[1.2-3.3]$ & 0.0121 \\
\hline High ( $\geq 200$ mg/dL) vs. normal (<150 mg/dL) & $2.3[1.2-4.2]$ & 0.0074 & $3.1[1.7-5.6]$ & 0.0003 & $1.3[0.5-3.7]$ & 0.5616 & $3.3[1.9-5.5]$ & $<0.0001$ \\
\hline \multicolumn{9}{|l|}{ High-sensitivity CRP } \\
\hline $\begin{array}{l}\text { Mild inflammation (1-3 mg/dL) vs. normal (<1 } \\
\mathrm{mg} / \mathrm{dL} \text { ) }\end{array}$ & $1.8[1.0-3.2]$ & 0.0562 & $1.4[0.8-2.6]$ & 0.2520 & $1.2[0.7-2.0]$ & 0.5222 & $1.1[0.6-2.0]$ & 0.8330 \\
\hline $\begin{array}{l}\text { Significant inflammation (3-10 mg/dL) vs. normal } \\
(<1 \mathrm{mg} / \mathrm{dL})\end{array}$ & $1.4[0.7-3.0]$ & 0.3518 & $1.5[0.7-3.5]$ & 0.3001 & $1.0[0.5-1.9]$ & 0.9679 & $1.8[0.8-4.2]$ & 0.1633 \\
\hline $\begin{array}{l}\text { High significant inflammation ( } \geq 10 \mathrm{mg} / \mathrm{dL} \text { ) vs. } \\
\text { normal }\end{array}$ & $1.1[0.5-2.4]$ & 0.7610 & $2.2[0.7-6.9]$ & 0.1900 & $1.2[0.6-2.4]$ & 0.5629 & $2.5[0.9-6.7]$ & 0.0697 \\
\hline \multicolumn{9}{|l|}{ Aspartate aminotransferase (AST) } \\
\hline Elevated (>40 U/L) vs. normal ( $\leq 40 \mathrm{U} / \mathrm{L})$ & $1.0[0.1-8.2]$ & 0.9759 & $1.3[0.6-2.8]$ & 0.5321 & $4.2[1.0-17.5]$ & 0.0485 & $5.4[1.3-22.5]$ & 0.0193 \\
\hline \multicolumn{9}{|l|}{ Alanine aminotransferase (ALT) } \\
\hline Elevated (>56 U/L) vs. normal ( $\leq 56 \mathrm{U} / \mathrm{L})$ & $2.5[0.6-11.5]$ & 0.2292 & $3.0[1.1-8.3]$ & 0.0389 & $0.1[0.0-0.8]$ & 0.0285 & $0.2[0.0-1.1]$ & 0.0669 \\
\hline \multicolumn{9}{|l|}{ Hemoglobin A1c (HbA1c) } \\
\hline Pre-diabetes vs. healthy (<5.7\%) & $1.5[0.9-2.3]$ & 0.1244 & $2.7[1.7-4.4]$ & $<0.0001$ & $1.2[0.7-2.2]$ & 0.4679 & $2.2[1.3-3.5]$ & 0.0019 \\
\hline Diabetes ( $\geq 6.5 \%$ ) vs. healthy (<5.7\%) & $1.3[0.5-3.3]$ & 0.6118 & $3.8[1.4-10.5]$ & 0.0097 & $2.0[1.0-3.9]$ & 0.0428 & $7.0[3.8-13.0]$ & $<0.0001$ \\
\hline
\end{tabular}


TABLE 7 | Adjusted odds ratio (AOR) and 95\% confidence interval (Cl) of the association between NAFLD stage and race/ethnicity for each gender group.

\begin{tabular}{|c|c|c|c|c|c|c|}
\hline \multirow{2}{*}{$\begin{array}{l}\text { Reference: normal/mild NAFLD } \\
\text { Race/ethnicity }\end{array}$} & \multicolumn{2}{|c|}{ Male } & \multicolumn{2}{|c|}{ Female no menopause } & \multicolumn{2}{|c|}{ Female menopause no hormone } \\
\hline & Moderate & Severe & Moderate & Severe & Moderate & Severe \\
\hline Non-Hispanic White & Ref & Ref & Ref & Ref & Ref & Ref \\
\hline Mexican American & $4.5[1.7-11.7]$ & $5.0[2.1-12.0]$ & $0.8[0.2-3.0]$ & $2.3[0.6-8.2]$ & $2.7[0.2-50.0]$ & $2.5[0.2-34.4]$ \\
\hline Other Hispanic & $1.2[0.5-2.6]$ & $0.7[0.3-2.2]$ & $0.6[0.1-2.3]$ & $1.8[0.7-4.8]$ & $0.2[0.0-4.7]$ & $0.4[0.0-12.2]$ \\
\hline Non-Hispanic Black & $1.0[0.5-1.9]$ & $0.5[0.3-0.9]$ & $0.7[0.4-1.2]$ & $0.5[0.3-0.9]$ & $0.7[0.2-1.9]$ & $0.6[0.2-1.7]$ \\
\hline Other race & $2.3[0.9-6.0]$ & $1.3[0.7-2.6]$ & $1.0[0.5-2.3]$ & $0.8[0.3-2.5]$ & $3.6[1.0-13.2]$ & $2.0[0.5-7.8]$ \\
\hline
\end{tabular}

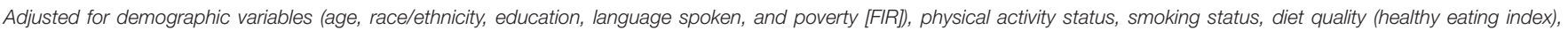

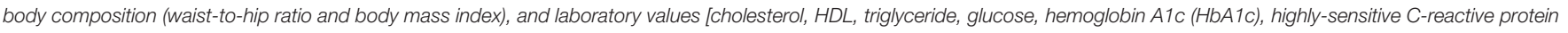
(hSCRP), AST, and ALT].

Bold $=$ statistically significant at $p<0.05$.

The study found positive associations between moderate NAFLD and older age (50+ years old), high-risk waist-to-hip ratio, BMI (overweight and obese), and high serum triglyceride. Additionally, there were strong associations between severe NAFLD and age of 50-64, high waist-to-hip ratio, BMI, highdensity lipoproteins, serum triglycerides, and HbAlc. The identified risk factors for NAFLD are consistent with other studies that found that an increased risk of NAFLD is associated with obesity, age, and other metabolic and cardiovascular diseases (13-15).

Importantly, the associations were modified when we stratified by sex. The increased odds of NAFLD observed for Mexican Americans and increased ALT levels was only present for males. Increases in odds observed for low levels of HDL and high levels of AST were observed only in females. When examining age-adjusted prevalence of NAFLD by stage, males had the highest prevalence of severe NAFLD followed by premenopausal women, followed by menopausal women without hormone therapy. Women on hormone therapy had a prevalence of severe NAFLD that was similar to that of males, although the sample size for this analysis was low. In the multivariable adjusted model, menopausal status did not affect the association between NAFLD and race/ethnicity, likely attributed to reduction in female hormones such as estrogen (29). Furthermore, the differences in risk factors related to gender suggests that the etiology or pathogenesis of NAFLD differ between the sexes. Previous studies had similarly found that in the adult population, men have a higher prevalence of NAFLD compared to women (22-42\% in men vs. $13-24 \%$ in women) $(15,30,31)$. In the Mexican pediatric population, NAFLD was more frequent in boys than girls $(24.51 \%$ in boys vs. $11.96 \%$ in girls) (32). Previous studies based on NHANES III data found NAFLD is significantly more prevalent in men than in women $(23,33-$ 38). Using NHANES 2017-2018 data, several studies saw that men had higher prevalence than women in both non-Hispanic white and Hispanic populations (39-41). Several factors may contribute to why we saw slightly different results, including that we did not combine Mexican Americans with other Hispanics; we characterized female sex by menopausal status; we were able to differentiate NAFLD from alcoholic fatty liver due to the availability of alcohol consumption data; and the CAP score cutoffs used to classify hepatic steatosis differed. Importantly, we also identified NAFLD related risk factors that differed by sex, which previous studies have not done. When examining reproductive-age groups, previous reports found that in the postmenopausal age, prevalence of NAFLD in men and women is comparable, whereas women in the premenopausal age have a lower prevalence compared to men $(15,30,42,43)$. The findings are consistent with our results, showing males had higher odds of NAFLD relative to non-menopausal but not menopausal females. There is evidence for gender differences in metabolic, inflammatory, and hormonal pathways that contribute to the differences observed in NAFLD (29-31). It will be important to investigate these differences further, with particular attention to differences by race/ethnicity.

\section{Strengths}

First, the current study uses data from the most recently released 2017/2018 cycle of NHANES, making the analysis more relevant in determining current prevalence and risk factors. Second, the data are a large national representative sample of the noninstitutionalized population in the US. Third, FibroScan ${ }^{\circledR}$, the method used to detect hepatic steatosis in the 2017/2018 cycle is more sensitive than some of the other methods used such as liver enzymes or CT, although it is less sensitive than other methods like magnetic resonance spectroscopy (44). Fourth, the new data categorize Mexican Americans separately from Americans of other Hispanic background, revealing an important dissociation of the prevalence in Mexican Americans compared to other Hispanic populations.

\section{Limitations}

One limitation of our study is that the NHANES data are cross-sectional, so we cannot determine the causal direction between NAFLD and its associated factors. Additionally, some variables, such as smoking, physical activity, menopausal status and hormone replacement were collected by self-report, so these estimates are prone to some recall bias. Numbers in the menopausal group on hormone replacement were low $(n=$ 110 ), so a lack of finding in the AOR of NAFLD in menopausal 
women not taking hormone therapy compared to those taking hormone therapy may be due to limited power. Although we controlled for major confounders and robust associations, it is possible that other unknown confounders could account for the associations found.

\section{Conclusions}

In conclusion, this study shows that the racial/ethnic difference in NAFLD differs by gender, with Mexican American men being at higher risk to develop the most severe form of the disease. The results indicate a gender disparity in NAFLD among the Hispanic US population. Our results also serve as another demonstration that the Hispanic American population is genetically and culturally diverse and care should be taken to avoid generalizing across groups of different backgrounds and gender. Screening and interventions that specifically target Mexican American males are needed to increase awareness about NAFLD and its prevention.

\section{DATA AVAILABILITY STATEMENT}

The datasets presented in this study can be found in online repositories. The datasets analyzed for this study can be

\section{REFERENCES}

1. Rich NE, Oji S, Mufti AR, Browning JD, Parikh ND, Odewole M, et al. Racial and ethnic disparities in nonalcoholic fatty liver disease prevalence, severity, and outcomes in the united states: a systematic review and meta-analysis. Clin Gastroenterol Hepatol. (2018) 16:198-210.e2. doi: 10.1016/j.cgh.2017.09.041

2. Younossi ZM. Non-alcoholic fatty liver disease - a global public health perspective. J Hepatol. (2019) 70:531-44. doi: 10.1016/ j.jhep.2018.10.033

3. Ruhl CE, Everhart JE. Fatty liver indices in the multiethnic United States National Health and Nutrition Examination Survey. Aliment Pharmacol Ther. (2015) 41:65-76. doi: 10.1111/apt.13012

4. Westfall E, Jeske R, Bader AR. Nonalcoholic fatty liver disease: common questions and answers on diagnosis and management. Am Fam Phys. (2020) 102:603-12.

5. Wong RJ, Aguilar M, Cheung R, Perumpail RB, Harrison SA, Younossi ZM, et al. Nonalcoholic steatohepatitis is the second leading etiology of liver disease among adults awaiting liver transplantation in the United States. Gastroenterology. (2015) 148:547-5. doi: 10.1053/j.gastro.2014.11.039

6. Younossi ZM, Blissett D, Blissett R, Henry L, Stepanova M, Younossi $\mathrm{Y}$, et al. The economic and clinical burden of nonalcoholic fatty liver disease in the United States and Europe. Hepatology. (2016) 64:1577-86. doi: 10.1002/hep.28785

7. Alkhouri N, McCullough AJ. Noninvasive diagnosis of NASH and liver fibrosis within the spectrum of NAFLD. Gastroenterol Hepatol. (2012) 8:661-8.

8. Ratziu V, Voiculescu M, Poynard T. Touching some firm ground in the epidemiology of NASH. J Hepatol. (2012) 56:23-5. doi: 10.1016/j.jhep.2011.08.002

9. Pais R, Barritt AS, Calmus Y, Scatton O, Runge T, Lebray P, et al. NAFLD and liver transplantation: current burden and expected challenges. J Hepatol. (2016) 65:1245-57. doi: 10.1016/j.jhep.2016.07.033

10. Zoller H, Tilg H. Nonalcoholic fatty liver disease and hepatocellular carcinoma. Metab Clin Exp. (2016) 65:1151-60. doi: 10.1016/j.metabol.2016.01.010 found at the CDC https://www.cdc.gov/nchs/nhanes/index. htm.

\section{ETHICS STATEMENT}

The studies involving human participants were reviewed and approved by NCHS of the CDC. The patients/participants provided their written informed consent to participate in this study.

\section{AUTHOR CONTRIBUTIONS}

MS, KS, DP, and DK performed analysis. MS, KS, DP, DK, and DE drafted the manuscript. MS, KS, DP, DK, VP, AZ, DE, SN, and TF reviewed and edited the manuscript. All authors contributed to the article and approved the submitted version.

\section{FUNDING}

This study was supported by the National Institutes of Health (grants NIMHD R01MD012579, U54MD007598, S21MD000103, NIDA R24DA017298, and NCATS UL1TR000124).

11. Dixon JB, Bhathal PS, O’Brien PE. Nonalcoholic fatty liver disease: predictors of nonalcoholic steatohepatitis and liver fibrosis in the severely obese. Gastroenterology. (2001) 121:91-100. doi: 10.1053/gast.2001.25540

12. Pais R, Maurel T. Natural history of NAFLD. J Clin Med. (2021) 10:1161. doi: $10.3390 /$ jcm 10061161

13. Rinella ME. Nonalcoholic fatty liver disease: a systematic review. JAMA. (2015) 313:2263-73. doi: 10.1001/jama.2015.5370

14. Vernon G, Baranova A, Younossi ZM. Systematic review: the epidemiology and natural history of non-alcoholic fatty liver disease and non-alcoholic steatohepatitis in adults. Aliment Pharmacol Therap. (2011) 34:274-85. doi: 10.1111/j.1365-2036.2011.04724.x

15. Samji NS, Snell PD, Singal AK, Satapathy SK. Racial disparities in diagnosis and prognosis of nonalcoholic fatty liver disease. Clin Liver Dis. (2020) 16:66-72. doi: 10.1002/cld.948

16. Shaheen M, Pan D, Schrode KM, Kermah D, Puri V, Zarrinpar A, et al. Reassessment of the Hispanic disparity: hepatic steatosis is more prevalent in Mexican Americans than other Hispanics. Hepatol Commun. (2021). doi: 10.1002/hep4.1775

17. Browning MG, Khoraki J, DeAntonio JH, Mazzini G, Mangino MJ, Siddiqui MS, et al. Protective effect of black relative to white race against non-alcoholic fatty liver disease in patients with severe obesity, independent of type 2 diabetes. Int J Obes. (2018) 42:926-9. doi: 10.1038/ijo.2017.309

18. Romeo S, Kozlitina J, Xing C, Pertsemlidis A, Cox D, Pennacchio LA, et al. Genetic variation in PNPLA3 confers susceptibility to nonalcoholic fatty liver disease. Nat Genet. (2008) 40:1461-65. doi: 10.1038/ng.257

19. Kozlitina J, Smagris E, Stender S, Nordestgaard BG, Zhou HH, TybjærgHansen A, et al. Exome-wide association study identifies a TM6SF2 variant that confers susceptibility to nonalcoholic fatty liver disease. Nat Genet. (2014) 46:352-6. doi: 10.1038/ng.2901

20. Mancina RM, Dongiovanni P, Phetta S, Pingitore P, Meroni M, Rametta R, et al. The MBOAT7-TMC4 variant rs641738 increases risk of nonalcoholic fatty liver disease in individuals of European descent. Gastroenterology. (2016) 150:1219-30.e6. doi: 10.1053/j.gastro.2016.01.032

21. Sherif ZA, Saeed A, Ghavimi S, Nouraie S-M, Laiyemo AO, Brim $\mathrm{H}$, et al. Global epidemiology of nonalcoholic fatty liver disease and 
perspectives on us minority populations. Digest Dis Sci. (2016) 61:1214-25. doi: 10.1007/s10620-016-4143-0

22. Nguyen GC, Segev DL, Thuluvath PJ. Racial disparities in the management of hospitalized patients with cirrhosis and complications of portal hypertension: a national study. Hepatology. (2007) 45:1282-9. doi: 10.1002/hep.21580

23. Harrison SA, Torgerson S, Hayashi PH. The natural history of nonalcoholic fatty liver disease: a clinical histopathological study. Am J Gastroenterol. (2003) 98:2042-7. doi: 10.1111/j.1572-0241.2003.07659.x

24. European Association for the Study of the Liver (EASL), European Association for the Study of Diabetes (EASD), and European Association for the Study of Obesity (EASO). EASL-EASD-EASO clinical practice guidelines for the management of non-alcoholic fatty liver disease. J Hepatol. (2016) 64:1388-402. doi: 10.1016/j.jhep.2015.11.004

25. Pan JJ, Fallon MB. Gender and racial differences in nonalcoholic fatty liver disease. World J Hepatol. (2014) 6:274-83. doi: 10.4254/wjh.v6.i5.274

26. NASH Clinical Research Network, Bass NM, Brunt EM, Clark JM, Diehl AM, Hoofnagle JH, et al. Clinical, laboratory and histological associations in adults with nonalcoholic fatty liver disease. Hepatology. (2010) 52:913-24. doi: 10.1002/hep.23784

27. Browning, JD, Szczepaniak LS, Dobbins R, Nuremberg P, Horton JD, Cohen JC, et al. Prevalence of hepatic steatosis in an urban population in the United States: impact of ethnicity. Hepatology. (2004) 40:1387-95. doi: 10.1002/hep.20466

28. Kallwitz ER, Daviglus ML, Allison MA, Emory KT, Zhao L, Kuniholm $\mathrm{MH}$, et al. Prevalence of suspected nonalcoholic fatty liver disease in Hispanic/Latino individuals differs by heritage. Clin Gastroenterol Hepatol. (2015) 13:569-76. doi: 10.1016/j.cgh.2014.08.037

29. Della Torre S. Non-alcoholic fatty liver disease as a canonical example of metabolic inflammatory-based liver disease showing a sex-specific prevalence: relevance of estrogen signaling. Front Endocrinol. (2020) 11:572490. doi: $10.3389 /$ fendo.2020.572490

30. Ministrini S, Montecucco F, Sahebkar A, Carbone F. Macrophages in the pathophysiology of NAFLD: the role of sex differences. Eur J Clin Investig. (2020) 50:e13236. doi: 10.1111/eci.13236

31. Lonardo A, Nascimbeni F, Ballestri S, Fairweather D, Win S, Than TA, et al. Sex differences in nonalcoholic fatty liver disease: state of the art and identification of research gaps. Hepatology. (2019) 70:1457-69. doi: 10.1002/hep.30626

32. Salvoza NC, Giraudi PJ, Tiribelli C, Rosso N. Sex differences in non-alcoholic fatty liver disease: hints for future management of the disease. Explor Med. (2020) 1:51-74. doi: 10.37349/emed.2020.00005

33. Lonardo A, Suzuki A. Nonalcoholic fatty liver disease: does sex matter? Hepatobiliary Surg Nutr. (2019) 8:164-6. doi: 10.21037/hbsn.2018.12.04

34. Villanueva-Ortega E, Garcés-Hernández MJ, Herrera-Rosas A, LópezAlvarenga JC, Laresgoiti-Servitje E, Escobedo G, et al. Gender-specific differences in clinical and metabolic variables associated with NAFLD in a Mexican pediatric population. Ann Hepatol. (2019) 18:693-700. doi: 10.1016/j.aohep.2019.04.012

35. Ruhl CE, Everhart JE. Determinants of the association of overweight with elevated serum alanine aminotransferase activity in the United States. Gastroenterology. (2003) 124:71-9. doi: 10.1053/gast.2003.50004
36. Clark JM, Brancati FL, Diehl AM. The prevalence and etiology of elevated aminotransferase levels in the United States. Am J Gastroenterol. (2003) 98:960-7. doi: 10.1111/j.1572-0241.2003.07486.x

37. Lazo M, Hernaez R, Eberhardt MS, Bonekamp S, Kamel I, Guallar E, et al. Prevalence of nonalcoholic fatty liver disease in the United States: the Third National Health and Nutrition Examination Survey, 1988-1994. Am J Epidemiol. (2013) 178:38-45. doi: 10.1093/aje/kws448

38. Schneider AL, Lazo M, Selvin E, Clark JM. Racial differences in nonalcoholic fatty liver disease in the U.S. population. Obesity. (2014) 22:292-9. doi: 10.1002/oby.20426

39. Kim D, Cholankeril G, Loomba R, Ahmed A. Prevalence of fatty liver disease and fibrosis detected by transient elastography in adults in the United States, 2017-2018. Clin Gastroenterol Hepatol. (2021) 19:1499-501.e2. doi: 10.1016/j.cgh.2020.08.017

40. Zhang X, Heredia NI, Balakrishnan M, Thrift AP. Prevalence and factors associated with NAFLD detected by vibration controlled transient elastography among US adults: Results from NHANES 2017-2018. PLoS ONE. (2021) 16:e0252164. doi: 10.1371/journal.pone.0252164

41. Zhou K, Dodge JL, Yuan L, Terrault NA. Metabolic risk profiles for hepatic steatosis differ by race/ethnicity: an elastography-based study of US adults. Dig Dis Sci. (2021) 1-16. doi: 10.1007/s10620-021-07124-w

42. Hamaguchi M, Kojima T, Ohbora A, Takeda N, Fukui M, Kato T. Aging is a risk factor of nonalcoholic fatty liver disease in premenopausal women. World J Gastroenterol. (2012) 18:237-43. doi: 10.3748/wjg.v18.i3.237

43. Wang Z, Xu M, Hu Z, Shrestha UK. Prevalence of nonalcoholic fatty liver disease and its metabolic risk factors in women of different ages and body mass index. Menopause. (2015) 22:667-73. doi: 10.1097/GME.0000000000000352

44. Bohte AE, van Werven JR, Bipat S, Stoker J. The diagnostic accuracy of US, CT, MRI and $1 \mathrm{H}$-MRS for the evaluation of hepatic steatosis compared with liver biopsy: a meta-analysis. Eur Radiol. (2011) 21:87-97. doi: 10.1007/s00330-010-1905-5

Conflict of Interest: The authors declare that the research was conducted in the absence of any commercial or financial relationships that could be construed as a potential conflict of interest.

Publisher's Note: All claims expressed in this article are solely those of the authors and do not necessarily represent those of their affiliated organizations, or those of the publisher, the editors and the reviewers. Any product that may be evaluated in this article, or claim that may be made by its manufacturer, is not guaranteed or endorsed by the publisher.

Copyright (c) 2021 Shaheen, Schrode, Pan, Kermah, Puri, Zarrinpar, Elisha, Najjar and Friedman. This is an open-access article distributed under the terms of the Creative Commons Attribution License (CC BY). The use, distribution or reproduction in other forums is permitted, provided the original author(s) and the copyright owner(s) are credited and that the original publication in this journal is cited, in accordance with accepted academic practice. No use, distribution or reproduction is permitted which does not comply with these terms. 